\title{
Modeling human adaptive immune responses with tonsil organoids
}

\author{
Lisa E. Wagar ${ }^{1,15}$, Ameen Salahudeen ${ }^{2,16}$, Christian M. Constantz ${ }^{3}$, Ben S. Wendel ${ }^{1}$, Michael M. Lyons ${ }^{1}$, \\ Vamsee Mallajosyula ${ }^{3}$, Lauren P. Jatt ${ }^{3}{ }^{3}$, Julia Z. Adamska ${ }^{4,5}$, Lisa K. Blum ${ }^{4,5}$, Neha Gupta ${ }^{3}$, \\ Katherine J. L. Jackson ${ }^{6}$, Fan Yang ${ }^{6}$, Katharina Röltgen ${ }^{6}$, Krishna M. Roskin ${ }^{6}$, Kelly M. Blaine ${ }^{7}$, \\ Kara D. Meister ${ }^{8,9}$, Iram N. Ahmad ${ }^{8}$, Mario Cortese ${ }^{10}$, Emery G. Dora ${ }^{10}$, Sean N. Tucker ${ }^{10}$, \\ Anne I. Sperling7, Aarti Jain"1, D. Huw Davies"11, Philip L. Felgner"1, Gregory B. Hammer ${ }^{12}$, Peter S. Kim ${ }^{13}$, \\ William H. Robinson ${ }^{4,5}$, Scott D. Boyd ${ }^{6}$, Calvin J. Kuo $\mathbb{1}^{2}$ and Mark M. Davis $\mathbb{1}^{1,3,14 \bowtie}$
}

\begin{abstract}
Most of what we know about adaptive immunity has come from inbred mouse studies, using methods that are often difficult or impossible to confirm in humans. In addition, vaccine responses in mice are often poorly predictive of responses to those same vaccines in humans. Here we use human tonsils, readily available lymphoid organs, to develop a functional organotypic system that recapitulates key germinal center features in vitro, including the production of antigen-specific antibodies, somatic hypermutation and affinity maturation, plasmablast differentiation and class-switch recombination. We use this system to define the essential cellular components necessary to produce an influenza vaccine response. We also show that it can be used to evaluate humoral immune responses to two priming antigens, rabies vaccine and an adenovirus-based severe acute respiratory syndrome coronavirus 2 vaccine, and to assess the effects of different adjuvants. This system should prove useful for studying critical mechanisms underlying adaptive immunity in much greater depth than previously possible and to rapidly test vaccine candidates and adjuvants in an entirely human system.
\end{abstract}

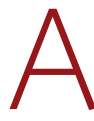
ntigen recognition by lymphocytes has been studied by immunologists since the discovery of antibodies and their specificities over a century ago ${ }^{1-4}$, followed by the more recent discovery of $\mathrm{T}$ cells and their antigen receptors in the $1960 \mathrm{~s}-1980 \mathrm{~s}^{5-7}$. The B cells that are responsible for forming a neutralizing antibody response develop within germinal centers (GCs) and extrafollicular regions in lymphoid organs $\mathrm{s}^{8-11}$. Upon antigen presentation by antigen-presenting cells (APCs) $)^{12-15}$, $\mathrm{T}$ follicular helper $\left(\mathrm{T}_{\mathrm{FH}}\right)$ cells, and a variety of hematopoietic and non-hematopoietic cells interact and deliver signals to GC B cells for survival, proliferation, antibody affinity maturation, class-switch recombination and differentiation ${ }^{16,17}$. Almost all of these interactions have been elucidated through in vivo studies of inbred mice. While these have produced a wealth of important information ${ }^{8,18-20}$, the lack of a system that replicates the essential features of human adaptive immunity, such as affinity maturation and class switching, and the effects of adjuvants, leaves many mechanistic aspects inaccessible. This is especially important for vaccine testing since many candidates that worked in animal models ultimately fail in human trials ${ }^{21-24}$, suggesting that genetic and environmental differences among species are important considerations in vaccine development.
Many in vitro systems rely on isolation of small chunks or slices of a tissue sample to prepare explant cultures ${ }^{25-31}$. Explant methods, although useful for studying individual aspects of infection and immunity ${ }^{30,32-34}$, typically do not maintain cell composition for very long (3-4d), nor do they capture all of the features of an adaptive immune response. Although bioreactor, chip-based and other specialized in vitro differentiation systems show promise, they too have not been able to replicate the complexities of adaptive immunity. We decided to take advantage of the widespread availability of human tonsils, lymphoid organs that are easily procured from tonsillectomy surgeries as discarded tissue, to develop an accessible system that replicates an antigen-specific adaptive immune response to a vaccine and supports key aspects of adaptive immunity.

\section{Results}

Preparation of immune organoids from tonsils and other lymphoid tissues. Over 20 years ago, Owen and Jenkinson demonstrated that dissociated murine thymic cells could reassociate in culture and recapitulate major aspects of $\mathrm{T}$ cell selection ${ }^{35,36}$. We applied a similar approach to develop human tonsil cultures with dissociated cells that reaggregate in culture (Fig. 1a; see Supplementary

\footnotetext{
'Department of Microbiology and Immunology, Stanford University, Stanford, CA, USA. ${ }^{2}$ Department of Medicine, Division of Hematology, Stanford University School of Medicine, Stanford, CA, USA. ${ }^{3}$ nnstitute for Immunity, Transplantation and Infection, Stanford University, Stanford, CA, USA. ${ }^{4}$ Department of Medicine, Division of Immunology and Rheumatology, Stanford University School of Medicine, Stanford, CA, USA. ${ }^{5}$ Veterans Affairs Palo Alto Healthcare System, Palo Alto, CA, USA. ' $D$ epartment of Pathology, Stanford University School of Medicine, Stanford, CA, USA. 'Department of Medicine, University of Chicago, Chicago, IL, USA. ${ }^{8}$ Department of Otolaryngology-Head \& Neck Surgery, Stanford University School of Medicine, Stanford, CA, USA. ${ }^{9}$ Aerodigestive and Airway Reconstruction Center, Lucile Packard Children's Hospital, Stanford, CA, USA. ${ }^{10}$ Vaxart, South San Francisco, CA, USA. "Vaccine Research and Development Center, Department of Physiology and Biophysics, University of California, Irvine, Irvine, CA, USA. ${ }^{12}$ Departments of Pediatrics and Anesthesiology, Perioperative and Pain Medicine, Stanford University School of Medicine, Stanford, CA, USA. ${ }^{13}$ Stanford ChEM-H, Department of Biochemistry and Chan Zuckerberg Biohub, Stanford University, Stanford, CA, USA. ${ }^{14}$ Howard Hughes Medical Institute, Stanford University, Stanford, CA, USA. ${ }^{15}$ Present address: Department of Physiology \& Biophysics, University of California, Irvine, Irvine, CA, USA. ${ }^{16}$ Present address: Department of Medicine, University of Illinois at Chicago, Chicago, IL, USA. $\varpi_{e}$-mail: mmdavis@stanford.edu
} 
Table 1 for tissue donor characteristics). For organoid preparation, frozen single-cell suspensions from tonsil tissues were thawed and plated at high density into the wells of permeable membrane plates (commonly known as Transwells) along with the antigen of interest. After several days in culture, reaggregated regions of clustered cells were visible (Fig. 1a). We assessed the cell composition of the reaggregated cultures after $7 \mathrm{~d}$ in the presence or absence of antigen and our optimized culture conditions (Methods) sustained appropriate tonsil cell composition (Fig. 1b). We used influenza vaccines and viruses as model antigens since much is already known about the features of the human influenza response in vivo. Upon stimulation with live attenuated influenza vaccine (LAIV), there were notable increases in B cell differentiation and a more structured culture morphology developed, suggesting additional activity in response to this immunogen.

We measured the capacity of organoid cultures to support B cell maturation and function upon LAIV stimulation (see Extended Data Fig. 1a for representative staining) and found that after $7 d$, plasmablast frequencies were significantly increased compared to unstimulated controls (Fig. $1 c ; n=15 ; P=0.0002$ ). Thirteen of the fifteen donors tested, ranging in age from 2-14 years, produced influenza-specific IgG antibodies in only the LAIV-stimulated samples $(P<0.0001)$. Of the two exceptions, one donor had a high response without stimulation and the other was a true nonresponder. The latter donor (a 3-year-old) may have been naive for influenza infection and had no reported influenza vaccination. The production of specific antibody was not a generic effect of stimulation, as total (that is, antigen agnostic) IgG was reduced by LAIV (Fig. 1d). We also performed enzyme-linked immune absorbent spot (ELISpot) analysis on day-7 cultures from five donors and found influenza-specific antibody-secreting cells (ASCs) ranging from $0.1-1.5 \%$ of total B cells (Fig. 1e; representative ELISpot data in Extended Data Fig. 1b). Almost no influenza-specific ASCs were identified in unstimulated tonsil cultures $(<0.02 \%$ of total B cells in all donors). The Transwell organoid culture strategy was superior to flat-bottom tissue culture plates of similar surface area for growing tonsil mononuclear cells (Extended Data Fig. 1c).

We also tested whether our organoid culture strategy could support human lung-draining lymph node and spleen samples (Fig. 1f). These organoids responded to both LAIV and wild-type A/California H1N1 influenza virus by producing influenza-specific antibodies at levels substantially above those of control cultures and equivalent to tonsil organoids.

Longitudinal analysis of tonsil cultures in response to LAIV. Given the evidence of B cell maturation and antibody secretion in organoid cultures stimulated with LAIV, we performed longitudinal analyses to observe the differentiation process over time. CD38 and CD27 expression patterns on B cells from LAIV-stimulated cultures clearly evolved (Fig. 2a), indicating an early transition to a pre-GC phenotype $\left(\mathrm{CD} 38^{+} \mathrm{CD} 27^{-}\right)$, and in two of three donors, this was followed by plasmablast differentiation (Fig. 2b). The frequency of plasmablasts typically correlated with the total amount of specific antibodies produced (Extended Data Fig. 1d). Changes in T cell phenotypes were also observed, including recovery of $\mathrm{T}_{\mathrm{FH}}\left(\mathrm{CXCR} 5^{+} \mathrm{CD} 4^{+}\right)$cells during culture (in both unstimulated and LAIV-stimulated organoids), as well as transient $\mathrm{CD}^{+}$and $\mathrm{CD}^{+} \mathrm{T}$ cell activation at day 7 in LAIV-stimulated cultures (Fig. 2c). Organoids prepared from only one of the over 50 donors tested (IMD012) did not produce influenza-specific antibodies (Fig. 2d). With four additional donors, we confirmed that influenza-specific antibodies are produced with the expected kinetics (between days 5 and 10). We also gauged the ability of these cultures to produce neutralizing antibodies; in the two strains tested (of the four LAIV strains), vaccine-stimulated culture supernatants showed neutralizing capabilities, while unstimulated controls did not (Fig. 2e).

We also assessed the $\mathrm{T}$ cell response in HLA-A2 ${ }^{+}$donors using class I tetramers targeting the immunodominant influenza M1 specificity (Fig. 2f). Three of the four donors tested showed a two- to ten-fold expansion of M1-specific $\mathrm{CD}^{+} \mathrm{T}$ cells by day 7 after stimulation with LAIV. The one nonresponder had fewer M1-specific $\mathrm{CD}^{+} \mathrm{T}$ cells in their starting repertoire compared to the other donors, which may explain their poor $\mathrm{CD}^{+} \mathrm{T}$ cell response.

Spatial organization of tonsil organoids. During an adaptive immune response, peripheral lymphoid organs like the tonsils, lymph nodes and spleen develop GCs. B cells congregate in the GC area, whereas $\mathrm{T}$ cells are largely in outlying areas ${ }^{8,19}$. In the organoid cultures, we found GC-like structures with distinct B cell- and T cell-rich aggregates in both LAIV-stimulated and unstimulated cultures, starting around $48 \mathrm{~h}$ after culture initiation. These progressed to well-defined clusters by days 4-7, particularly in LAIV-treated cultures (Fig. 3a and Extended Data Fig. 2). We analyzed the embedded, frozen sections of three donors by fluorescent confocal microscopy and found evidence of light and dark zone organization, as detected by separation of CD83 and CXCR4 staining B cells, respectively, which are characteristic of GCs (Fig. 3a; additional representative images are available in Extended Data Figs. 2 and 3a).

We also performed a single-cell RNA-seq analysis along with DNA-barcoded antibodies using the BD Rhapsody platform to characterize the expression profiles of GC-phenotype B cells in organoid cultures. Dimensionality reduction (uniform manifold approximation and projection (UMAP)) was used to analyze GC $\mathrm{B}$ cells across different time points (Fig. 3b). Overall, GC B cells $\left(\mathrm{CD} 38^{+} \mathrm{CD} 27^{+}\right)$from the different days and time points clustered together, showing that their expression profiles were similar. The top overexpressed genes in GC B cells (compared to naive B cells) at day 5 of LAIV-stimulated cultures were related to antibody secretion (immunoglobulin heavy chains, light chains and J chain) and key $\mathrm{B}$ cell differentiation, proliferation and $\mathrm{B}$ cell receptor (BCR) signaling genes (XBP1, POU2AF1, S100A10 and PCNA). These were also the top differentially expressed genes between direct ex vivo GC and naive B cells. These data show that the cultures can functionally respond to antigen-specific stimulation, that light and dark zone phenotype B cells separate into distinct areas (Fig. 3a and Extended Data Fig. 3a) and that B cells with a GC phenotype have similar transcriptional profiles on day 0 and day 5 after culture (Fig. 3b), which collectively support the conclusion that these cultures can be considered organoids.

Diversity and maturation of hemagglutinin-specific $\mathbf{B}$ cells. We next examined whether activation-induced cytidine deaminase (AID), which is required for both somatic hypermutation and class switching, was expressed in these cultures. AID protein levels (Fig. 3c; see representative staining in Extended Data Fig. 3b) were significantly increased in pre-GC and GC B cells in 4-day-old organoid cultures (prevalence shown in Fig. 3c). We also single-cell sequenced BCRs from high-affinity A/California 2009 H1N1 hemagglutinin (HA)-specific plasmablasts (and other activated B cells) using a HA trimer construct ${ }^{37}$ on day- 0 and day-7 LAIV-stimulated cultures to evaluate affinity maturation and isotype switching. As previously reported in peripheral blood plasmablasts of influenza-vaccinated volunteers ${ }^{38}$, immunoglobulin heavy-chain variable gene cluster (IGHV) 3 and IGHV4 dominated the response (Fig. 3d; see Extended Data Fig. 4 for complete dataset). HA-specific BCRs from LAIV-stimulated organoids were quite diverse in both gene usage and isotype; numerous oligoclonal expansions were seen at day 7 , but these clones were distinct from those detected in day- 0 high-affinity $\mathrm{HA}^{+} \mathrm{B}$ cells. Clonally related BCR families were also found in LAIV-stimulated organoids, and in one of two 
$\mathbf{a}$
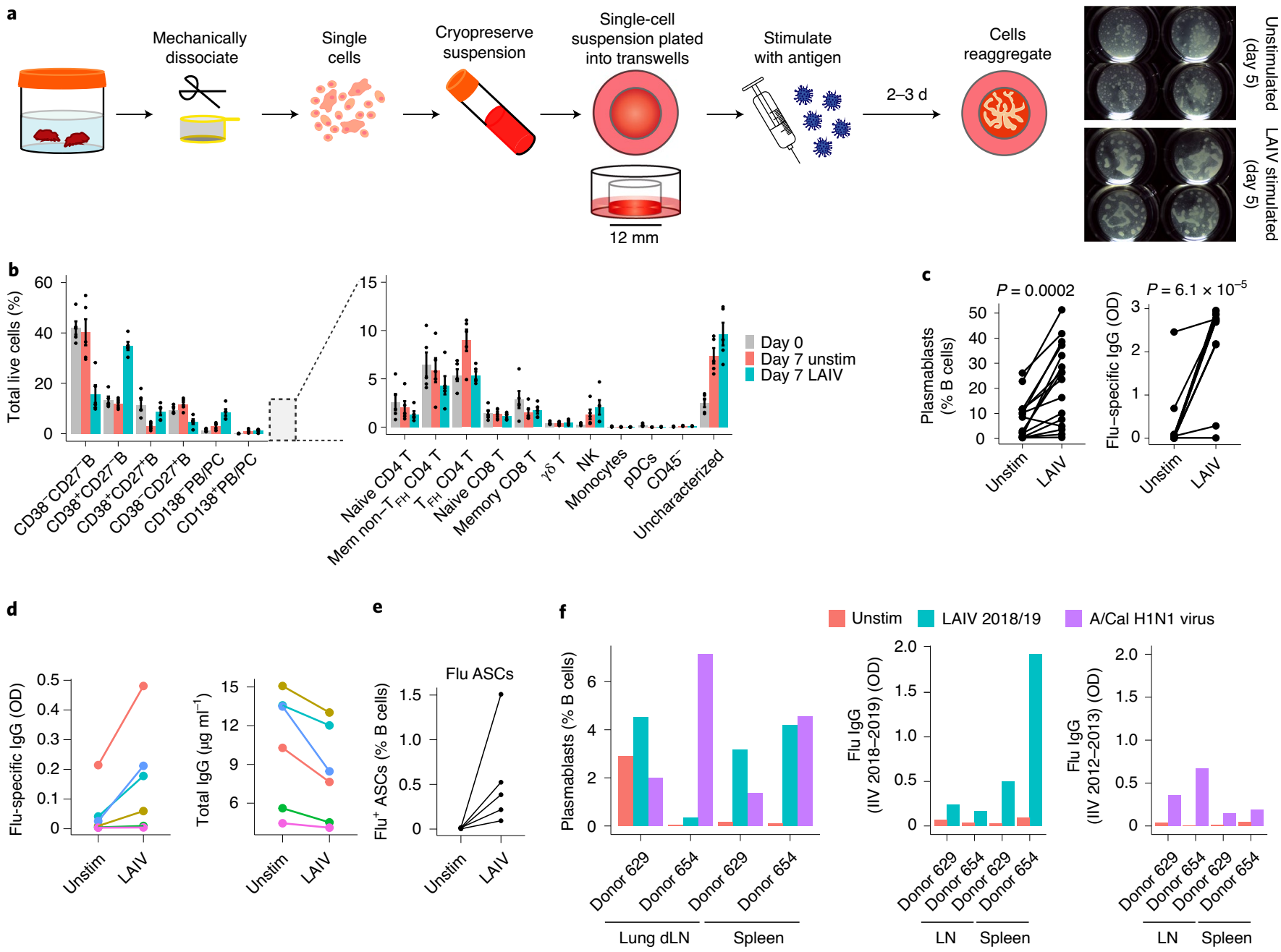

Fig. 1 Adaptive immune responses in tonsil organoids. a, Workflow for tissue disruption and culture preparation with representative stereoscope images from day-5 unstimulated or LAIV-stimulated replicate cultures. Light regions are areas of high cell density. $\mathbf{b}$, Cell composition of immune cell types in freshly isolated, frozen and revived day- 0 tonsil cells, day-7 unstimulated cultures and day-7 LAIV-stimulated cultures. Frequencies were determined by flow cytometry, and plotted values are the mean \pm s.e.m. $(n=5$ donors). PB/PCs, plasmablast/plasma cells; NK, natural killer. c, Plasmablast differentiation and specific antibody secretion from unstimulated and LAIV-stimulated organoids from day-7 cultures. Paired, two-sided Wilcoxon signed-rank tests were used to calculate $P$ values ( $n=15$ donors). OD, optical density. d, Antigen-specific antibodies and total IgG from day-7 unstimulated and LAIV-stimulated organoid cultures. Colors represent individual donors $(n=6)$. e, ASCs specific for influenza antigens from day-7 unstimulated and LAIV-stimulated cultures ( $n=5$ donors). f, Plasmablast frequencies and antibody secretion on day 14 from immune organoids derived from human lung-draining lymph nodes and spleens ( $n=2$ for each tissue). A/Cal, A/California H1N1 virus.

donors, we found clonal families with single amino acid changes, showing diversification within HA-specific lineages. Using bulk BCR sequencing of total non-naive B cells, we found lineages that began as the IgM isotype and class switched during organoid culture (mostly to IgG1 and IgA1), usually with additional mutations, by day 7 (Extended Data Fig. 5).

Affinity maturation in tonsil organoids. Affinity maturation is another key function of GCs. To evaluate this, we performed depletion experiments using FACS to eliminate preexisting high-affinity $\mathrm{HA}^{+} \mathrm{B}$ cells and also any non-naive $\mathrm{B}$ cells from the tonsil cell pool. Cultures were then prepared from the depleted cells, stimulated with LAIV, and stained again on day 10 to assess the development of new high-affinity $\mathrm{HA}^{+} \mathrm{B}$ cells (Fig. 3e and Extended Data Fig. 6). In three of four donors tested, $\mathrm{HA}^{+} \mathrm{B}$ cells reappeared in depleted cultures and influenza-specific secreted antibodies were detectable (Fig. 3f), demonstrating that affinity maturation can be directly observed in this system.
Next, we used IgH sequencing to analyze the BCR repertoire in naive versus affinity matured $B$ cells in response to LAIV stimulation. Here, we prepared cultures where naive, $\mathrm{HA}^{-} \mathrm{B}$ cells were the only B cell source (Fig. $4 \mathrm{a}$ and Methods). We used day- $0 \mathrm{HA}^{+} \mathrm{B}$ cells as the reference for high-affinity BCRs and to infer HA specificity. Organoid cultures were harvested on day 7 for bulk BCR repertoire sequencing (Fig. 4a). We compared the repertoires of these cultures to both the in vivo high-affinity $\mathrm{HA}^{+} \mathrm{B}$ cells from day- 0 tonsil cells that were depleted by FACS and the $\mathrm{HA}^{-}$naive $\mathrm{B}$ cells that went into culture preparations. Of the six donors tested, five secreted antibodies specific for vaccine components and four had detectable antibodies specific for the A/California $2009 \mathrm{H} 1 \mathrm{~N} 1 \mathrm{HA}$ protein on day 7 after stimulation (Fig. 4b), showing that we can derive HA-specific antibodies from naive B cells.

Somatic hypermutation was significantly increased in day-7 LAIV-stimulated cultures compared to unstimulated control cultures and was particularly enhanced where A/California $2009 \mathrm{H} 1 \mathrm{~N} 1$ HA antibodies were detected (IMD006, 013, 014 and 102; Fig. 4c). 

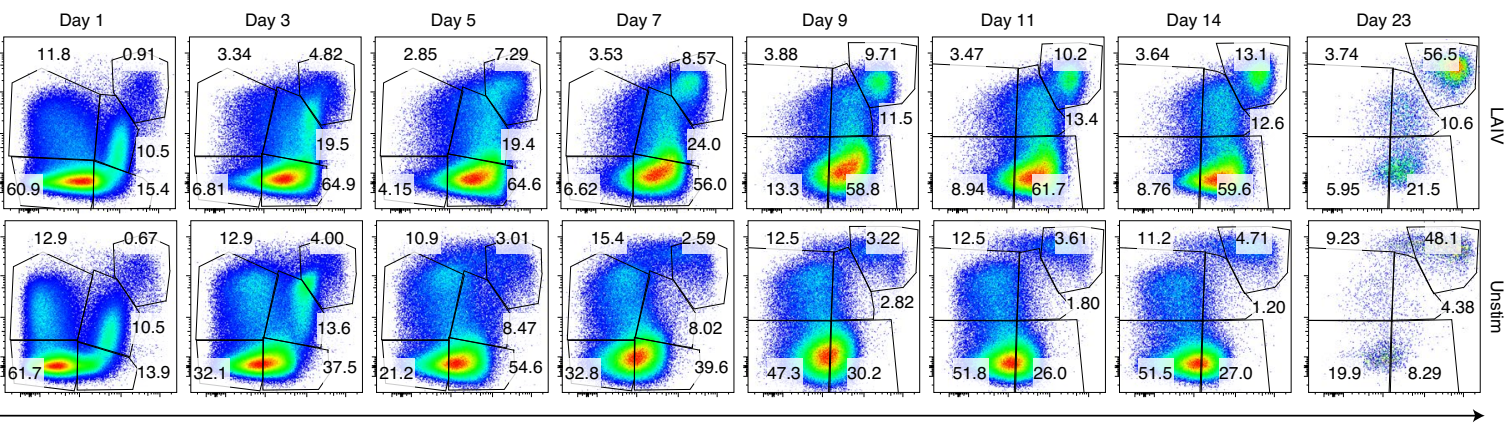

b

APC-CD38
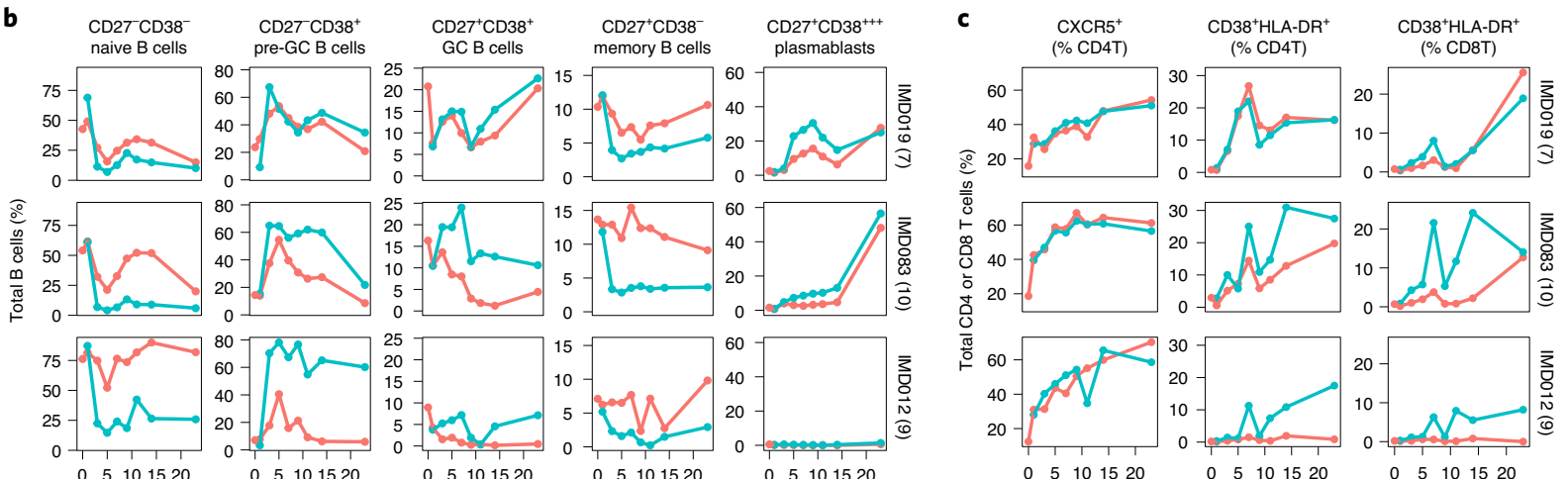

05101520

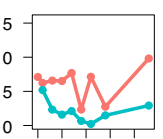
(d)
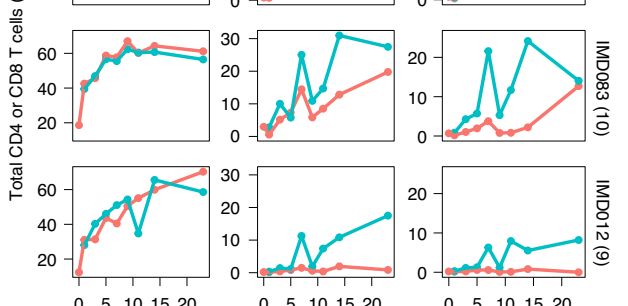

Unstimulated

LAIV stimulated

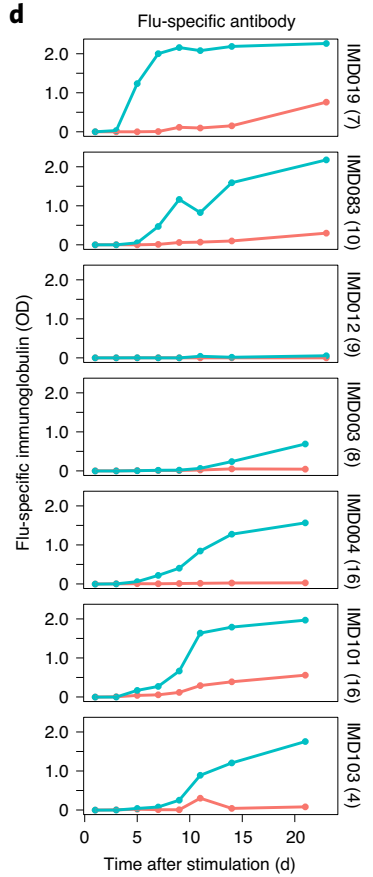

e
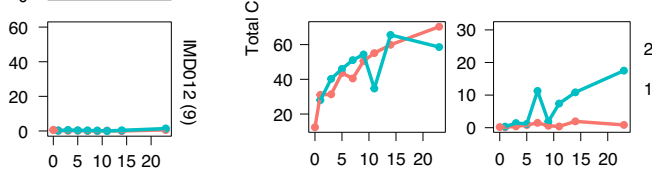

$\begin{array}{lllllll}0 & 5 & 101520 & 0 & 5 & 101520\end{array}$

Time after stimulation (d)
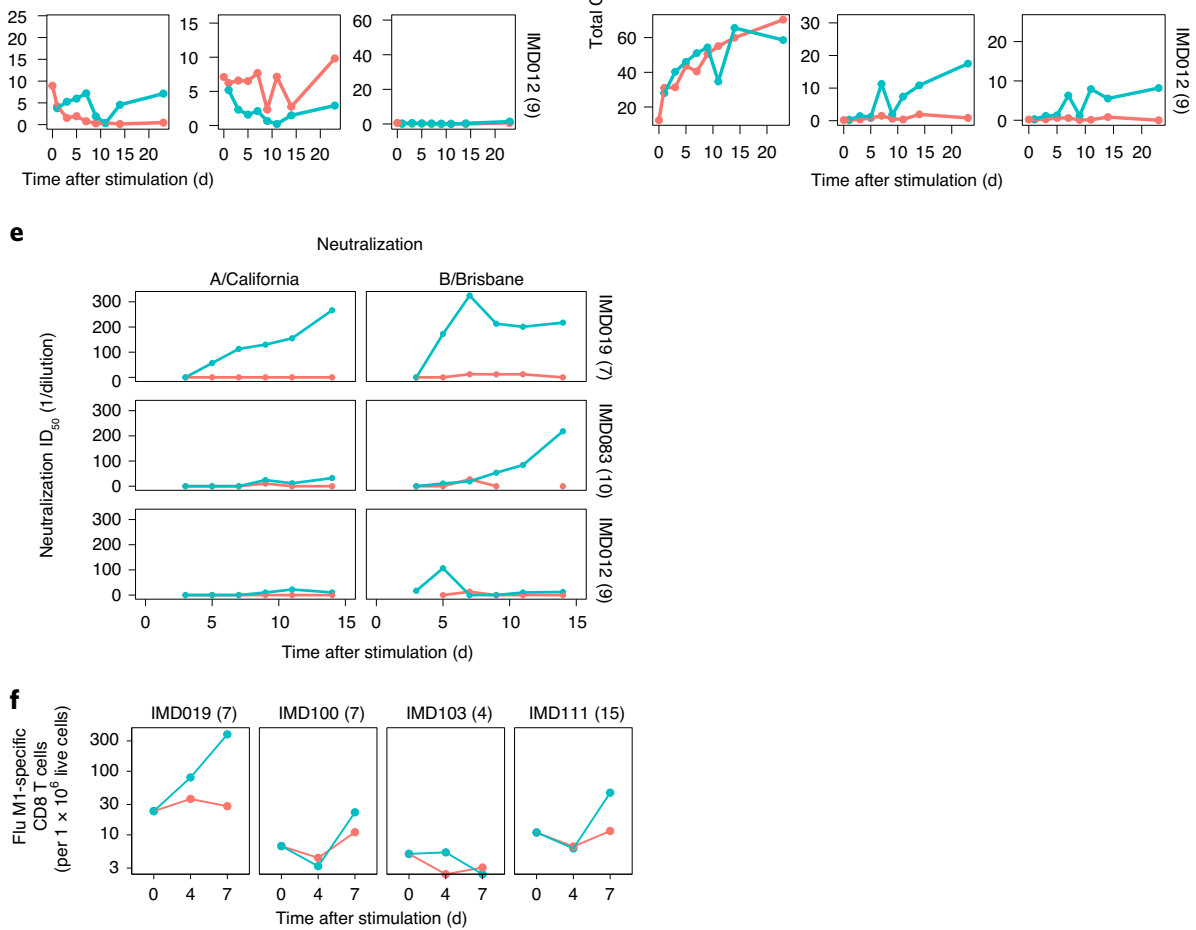

Fig. 2 | Longitudinal tracking of immune organoids reveals cellular and functional changes consistent with an adaptive response. a, Representative flow cytometry staining of B cell differentiation phenotypes in unstimulated and LAIV-stimulated cultures. Cells shown are pre-gated on total B cells $\left(C D 45^{+} C_{19}{ }^{+} C D 3^{-}\right) . \mathbf{b}, \mathbf{c}$, Quantitation of B cell (b) and T cell (c) phenotypes across time in unstimulated and LAIV-stimulated organoids.

d, Influenza-specific antibodies detected in culture supernatants. e, Neutralizing capacity of antibodies in culture supernatants against two of the four vaccine strains represented in the antigen stimulation (LAIV 2015-2016). Complete cellular and humoral data from $n=3$ donors (a-e) and longitudinal antibody detection data from an additional $n=4$ donors (d) are shown. $\mathbf{f}$, Influenza M1-specific CD8 ${ }^{+}$T cell responses in unstimulated and LAIV-stimulated tonsil organoids from four HLA-A2+ donors. Donor ages (in years) are shown in parentheses after donor IDs.

We also followed the development of BCR sequences likely (within one amino acid) to be specific for influenza strain A/California H1N1. In two of the six donors tested (IMD006 and IMD014), the $\mathrm{B}$ cell clones with inferred HA specificity for this strain were also larger than those without known HA specificity and this was dependent on vaccine stimulation (Fig. 4d). In these same donors, the average diversity was also increased, with more unique molecules per clonal lineage in HA-specific B cells from LAIV-stimulated 
a

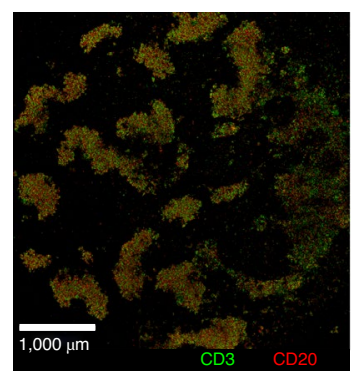

b
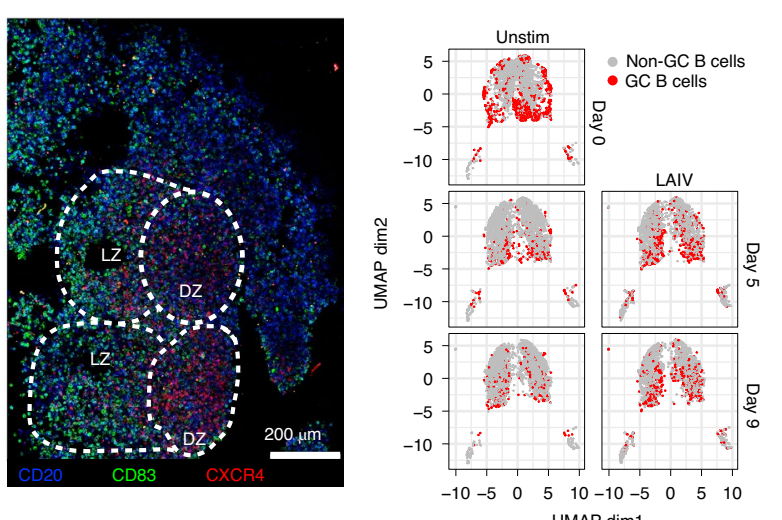
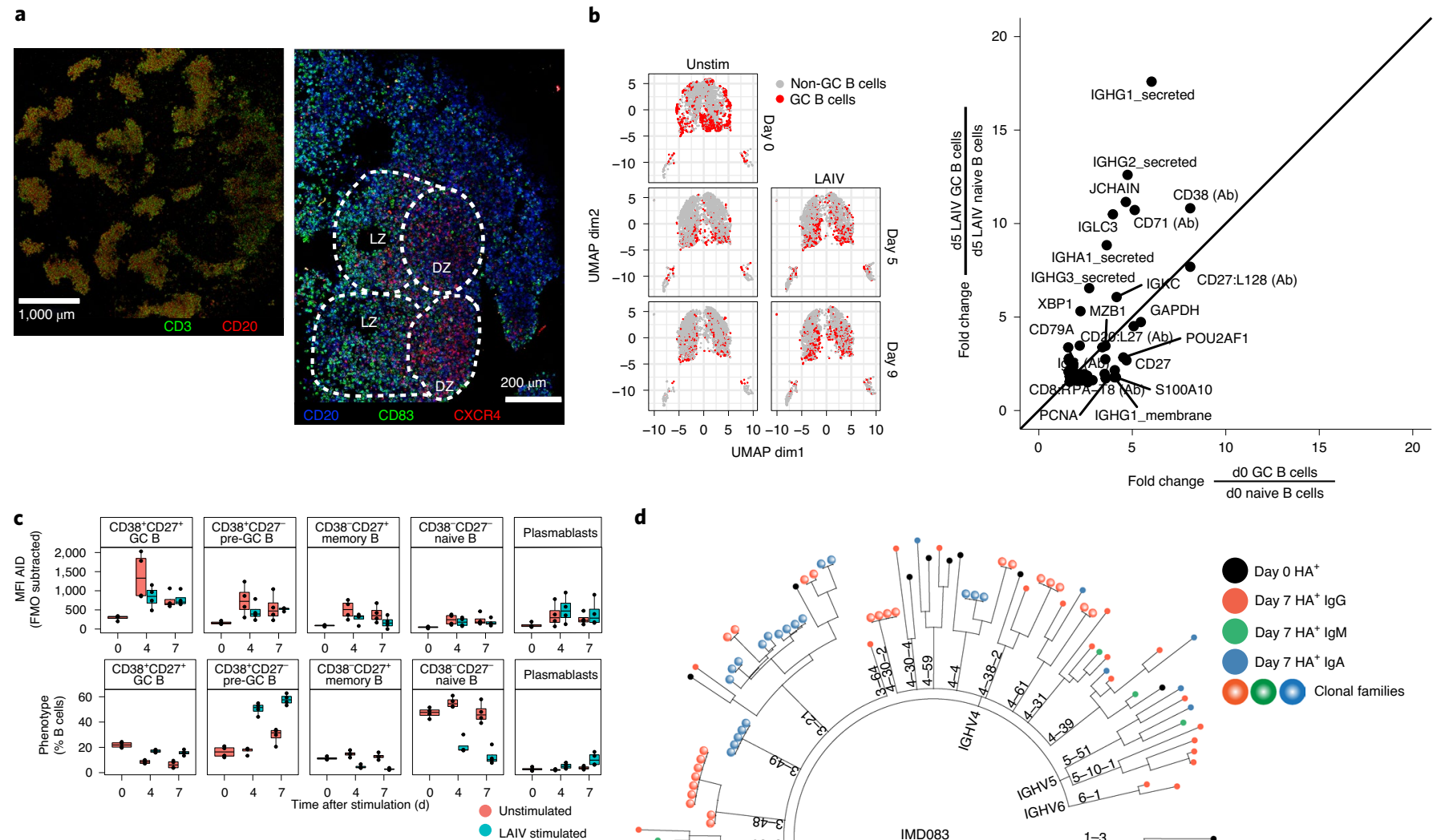

d
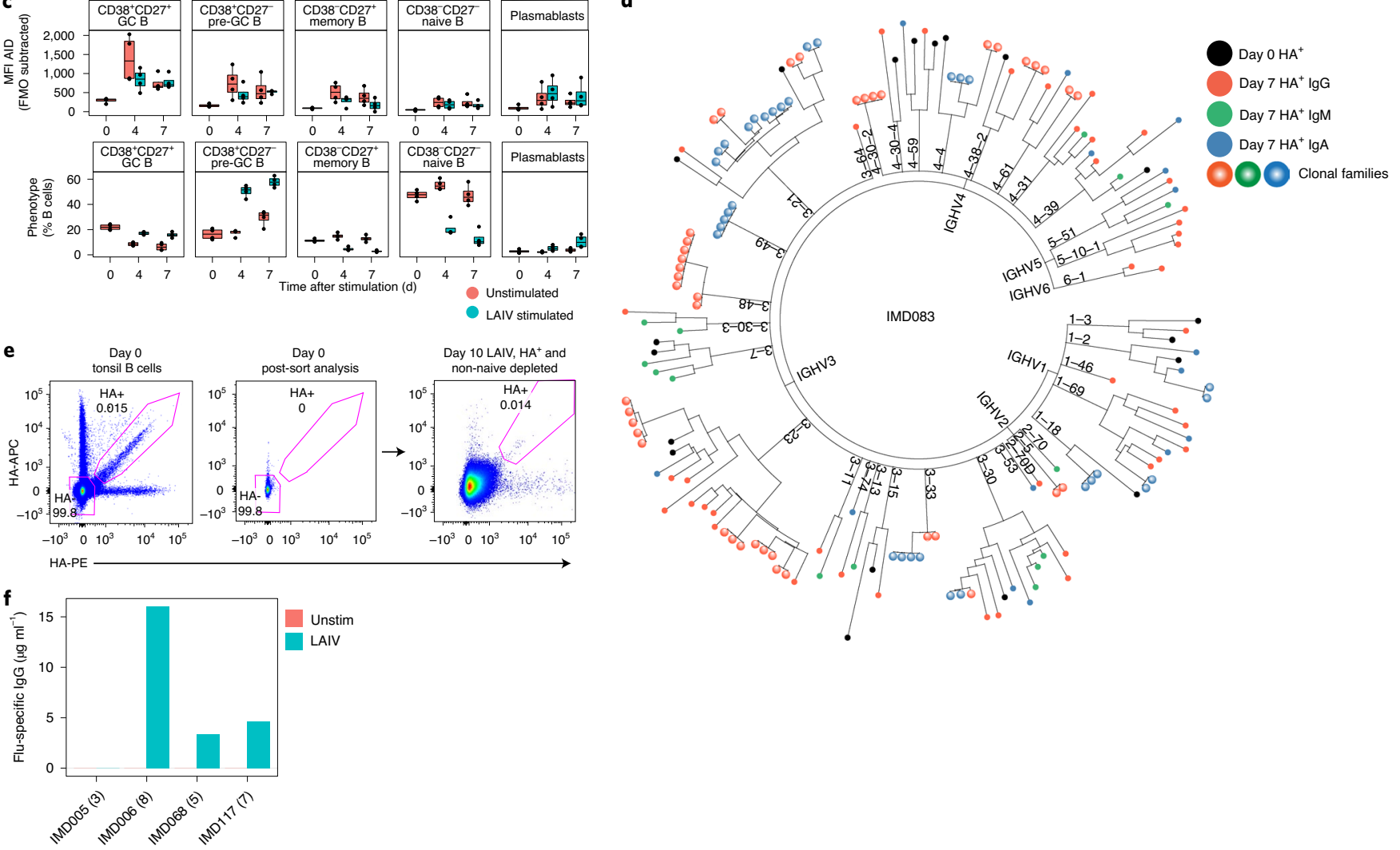

Fig. 3 | Diversity and maturation of the influenza response. a, Confocal microscopy images of a day-4 LAIV-stimulated organoid culture and B cell areas with light zone (LZ) and dark zone (DZ) organization. b. Single-cell RNA-seq of B cells from day-0 tonsil cells and organoid cultures from days 5 and 9 from one donor. The fold change values in genes and antibodies (Ab) highly expressed in GC compared to naive B cells (at least 1.5-fold increase) were plotted from day-0 (d0) B cells and day-5 (d5) LAIV-stimulated B cells. c, Median fluorescence intensity (MFI) for protein-level AID expression (top) and the relative proportions of $B$ cell subsets (bottom) on days 0,4 and 7 of organoid culture. Box plots show median values with hinges representing the first and third quartiles and whiskers representing the highest and lowest value that is within 1.5 times the interquartile range of the hinges ( $n=4$ donors). FMO, fluorescence minus one. d, Single-cell BCR sequencing of high-affinity A/California 2009 H1N1 HA-specific plasmablasts and GC B cells from one representative donor. High-affinity B cells specific for HA from day-O (black points) and day-7 LAIV-stimulated organoid cultures (green, blue and red points representing IgM, IgA and IgG isotypes, respectively) are shown. Clonal families are represented by open shading. e, Organoid cultures pre-depleted of high-affinity $\mathrm{A} /$ California $\mathrm{HA}^{+} \mathrm{B}$ cells and non-naive $\mathrm{B}$ cells produce new $\mathrm{HA}+\mathrm{B}$ cells. Effective depletion on day $\mathrm{O}$ of $\mathrm{HA}+\mathrm{B}$ cells and non-naive populations was confirmed by post-sort analysis, and new $\mathrm{HA}^{+} \mathrm{B}$ cells were detectable on day 10 . Data shown are representative plots from one of $n=4$ donors. $\mathbf{f}$, Influenza-specific antibodies were detected in depleted organoid cultures from three of $n=4$ donors tested. Donor ages (in years) are shown in parentheses after donor IDs.

cultures (Fig. 4d). We found other expanded and diverse lineages only in the vaccine-stimulated cultures, likely responding to other antigens or strains. We also found direct evidence for BCR sequence evolution to increased HA affinity (Fig. 4e). Here a root sequence with four nucleotide mutations from the germline heavy-chain sequence acquired an additional ten mutations to achieve an exact 
a

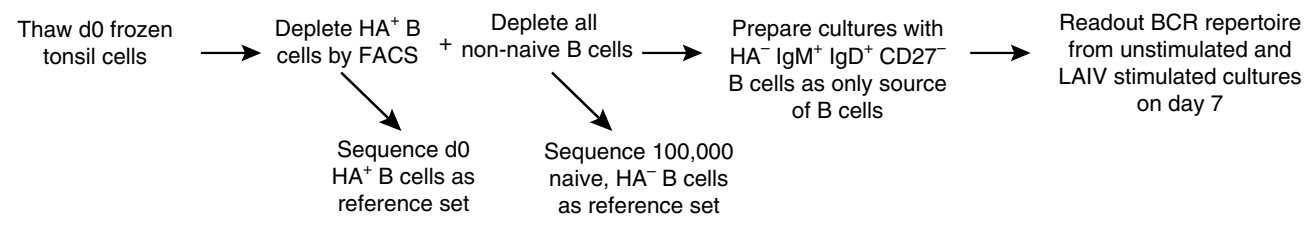

b

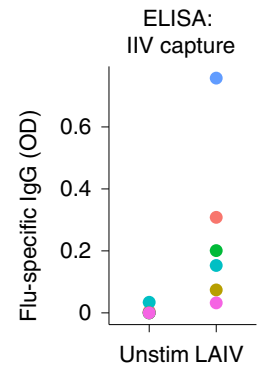

d

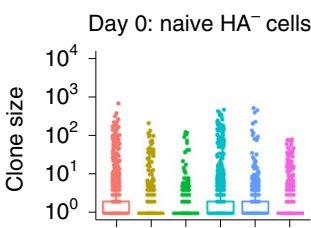

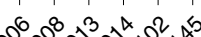

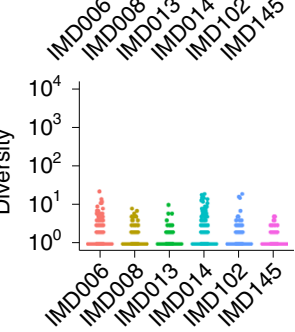

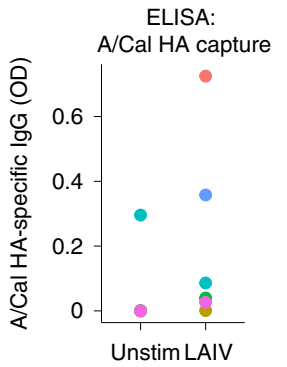

c
Day 0

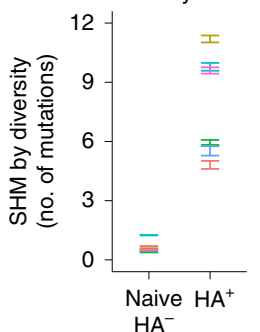

Day 7

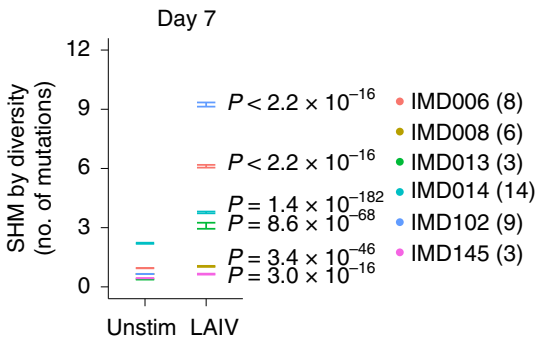

Day-7 organoids
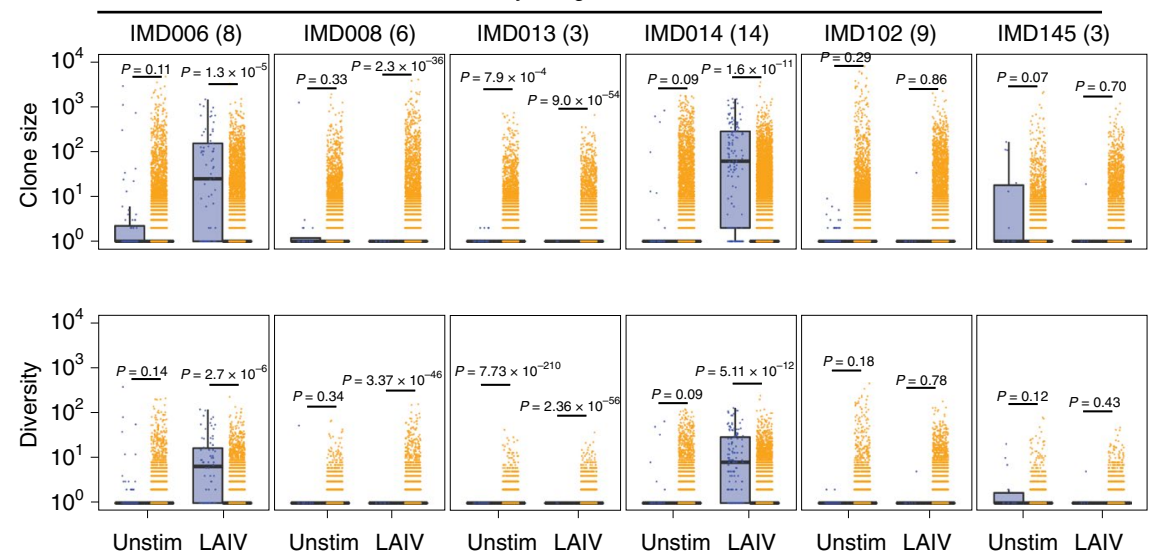

Inferred specificity

A/Cal HA

Not A/Cal HA

Fig. 4 | Somatic hypermutation and antigen-directed affinity maturation are supported in tonsil organoids. a, BCR repertoire analysis workflow on cultures prepared from tonsil cells depleted of high-affinity $\mathrm{HA}^{+}$and total non-naive B cells ( $n=6$ donors). $\mathbf{b}$, Influenza vaccine-specific and A/California 2009 H1N1 HA-specific antibodies after 7 d in unstimulated and LAIV-stimulated cultures. c, Somatic hypermutation as measured by the number of nucleotide mutations from the germline heavy-chain BCR sequences. Data are mean \pm s.e.m. and significance was calculated with two-sided Welch $t$-tests. d, Clone size (as measured by the number of RNA molecules per lineage) and diversity (as measured by the number of unique RNA molecules per lineage)

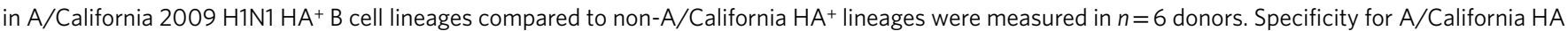
was inferred from the pool of known day- $0 \mathrm{HA}^{+} \mathrm{BCRs}$. Box plots show median values with hinges representing the first and third quartiles and whiskers representing the highest and lowest value that is within 1.5 times the interquartile range of the hinges. Each overlaid point represents an individual lineage. Significance values shown were calculated using two-sided Welch $t$-tests. Donor ages (in years) are shown in parentheses after donor IDs. e, Development of HA specificity in a BCR lineage from one donor. The size of the node represents the number of RNA molecules detected, the distance between nodes is proportional to the edit distance (nucleotide) between them, and the color reflects edit distance (amino acid) to the nearest known A/California HA-specific sequence, with light blue representing an exact match. The root sequence (56), major node (86) and a subset of the major node with lgG class switch (85) are highlighted. 
a

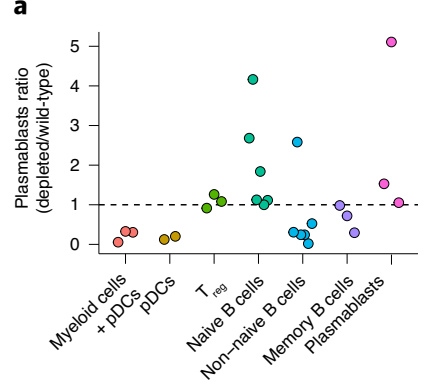

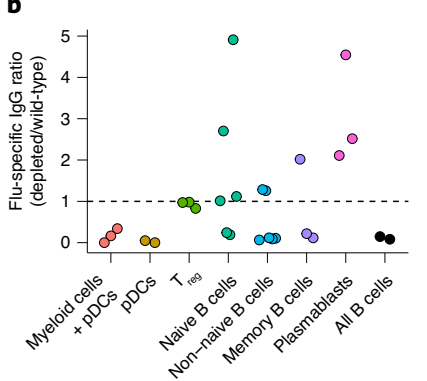

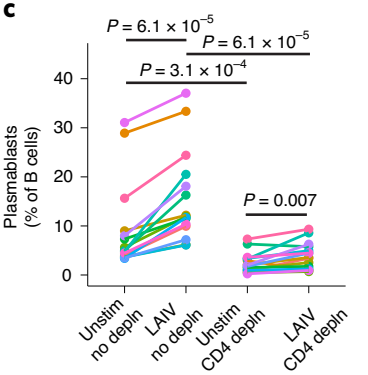

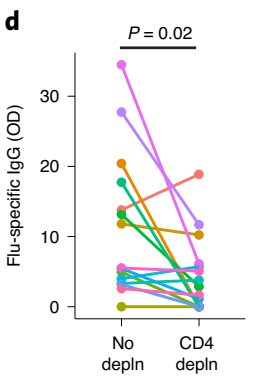

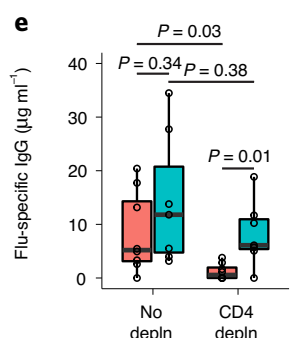

f
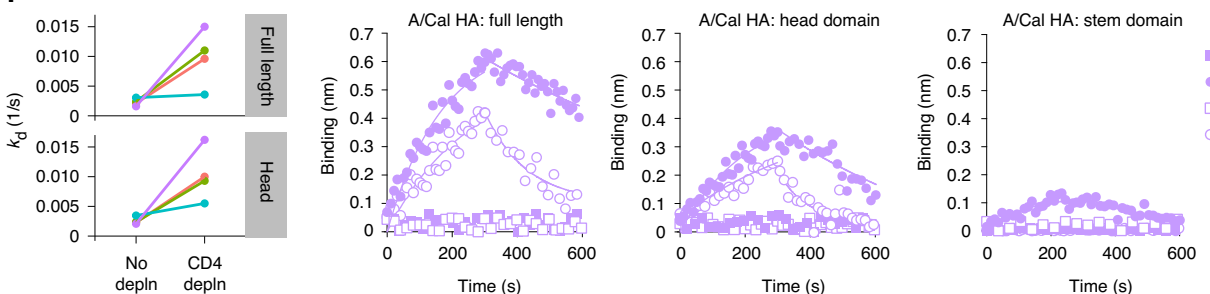

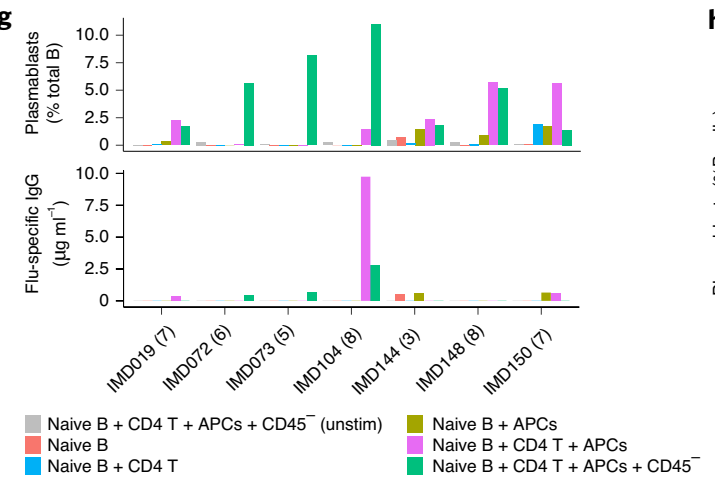

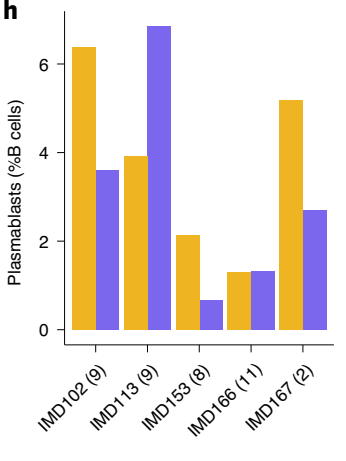

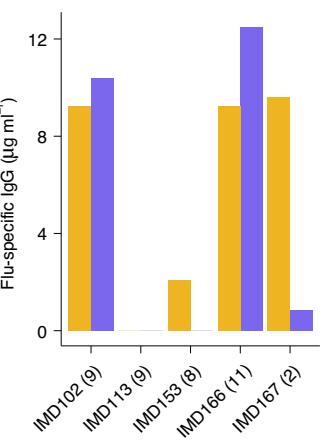

Unstim

LAIV

Unstim (CD4 depleted) LAIV (CD4 depleted)

Fig. 5 | Depletion studies reveal cell types required for plasmablast differentiation, specific antibody secretion and antibody affinity in organoid cultures. $\mathbf{a}, \mathbf{b}$, Effect of cell subset depletion on (a) plasmablast differentiation and (b) specific antibody secretion. Fold change in plasmablast frequency is relative to sorted cells reconstituted with the depleted cell type. Sort depletion experiments were performed on cells from $n=6$ (naive B cells and non-naive B cells), $n=2$ (total B cells and pDCs) or $n=3$ (myeloid cells $+p D C s$, regulatory $T\left(T_{\text {reg}}\right.$ ) cells, memory B cells and plasmablasts) donors. c,d, Effect of CD4 ${ }^{+}$depletion on (c) plasmablast differentiation and (d) antibody production after $7 \mathrm{~d}$ in organoid culture ( $n=15$ donors). Antibodies against influenza were not detected in unstimulated controls. Paired, two-sided Wilcoxon signed-rank tests were used to determine the significance values. e, Dependence on CD4+ cells for influenza-specific antibody secretion was associated with age. Intact or CD4+ depleted, LAIV-stimulated cultures were stratified into ages 5 years and under (red) or older than 5 (cyan). Box plots show the median values with hinges representing the first and third quartiles and whiskers representing the highest and lowest value that is within 1.5 times the interquartile range of the hinges. Unpaired, two-sided Mann-Whitney $U$ tests were used to calculate significance. f, Biolayer interferometry binding data from intact or CD4+-depleted cultures. Dissociation rates as measured by $k_{d}(1 / s)$ are shown for four donors and detailed binding traces from one representative donor. Data were collected from antibodies binding full-length A/California $2009 \mathrm{H} 1 \mathrm{~N} 1 \mathrm{HA}$ or head domain only. Stem-specific antibodies were detected in two donors but only under CD4-containing conditions. Colors are matched to donor data shown in c and $\mathbf{d}$. $\mathbf{g}$, Minimal cell requirements to support an influenza B cell response. Individual cell types were sorted and then recombined as shown to test for plasmablast differentiation and antibody secretion after $7 \mathrm{~d}$ of stimulation with LAIV ( $n=7$ donors). $\mathbf{h}$, Contribution of naive versus memory CD4+ T cells in B cell differentiation and influenza-specific antibody secretion under minimal cellular conditions. Naive B cells, APCs and CD45- cells were sorted and combined with equal numbers of either naive (CD45RA ${ }^{+} C D 27^{+}$) or memory (all non-naive) CD $4^{+} \mathrm{T}$ cells for $10 \mathrm{~d}$ in the presence of LAIV ( $n=5$ donors).

amino acid match to a known high-affinity HA specificity. Taken together, these data confirm that antigen-driven somatic hypermutation, affinity maturation and class switching are supported in tonsil organoid cultures.

Contributions of individual cell types to influenza responses. A major advantage of in vitro systems is the ability to define the essential components. Therefore, we depleted APC, T cell and B cell subsets and compared plasmablast differentiation (Fig. 5a,c) and antigen-specific antibody secretion (Fig. 5b,d) to wild-type organoids. Despite their low starting frequency $(0.1-0.5 \%$ of live), depleting myeloid and plasmacytoid dendritic cell (pDC) populations completely abrogated plasmablast differentiation (Fig. 5a) and depleting pDCs alone was sufficient to dramatically reduce the antibody response to LAIV (Fig. 5b). We were able to rescue the effect of pDC loss by adding type I interferon (IFN), showing that type I IFN production is the main pDC function (Extended Data Fig. 7a).

As expected, depleting $\mathrm{CD}^{+}$cells (Extended Data Fig. 7b) significantly reduced plasmablast differentiation, although some influenza-specific antibodies were still detectable (Fig. 5c,d). We analyzed the effect of donor age and found that $\mathrm{CD}_{4}^{+}$ $\mathrm{T}$ cell-depleted organoids from younger children were almost entirely unable to secrete influenza-specific antibody (Fig. 5e), in contrast to older donors who could make antibody responses. 

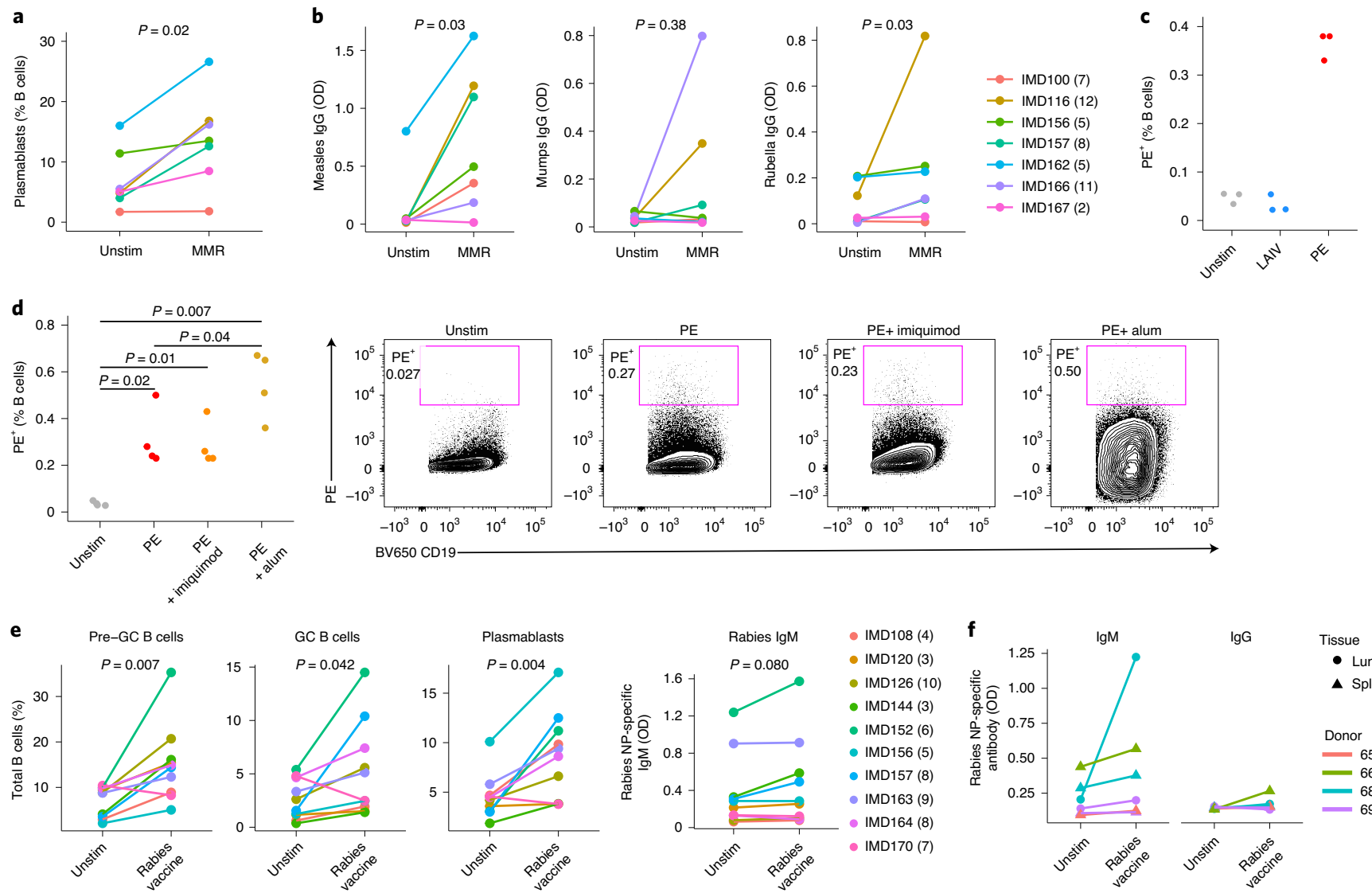

Tissue

- Lung dLN

A Spleen

Donor

g

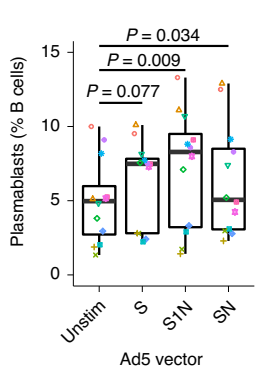

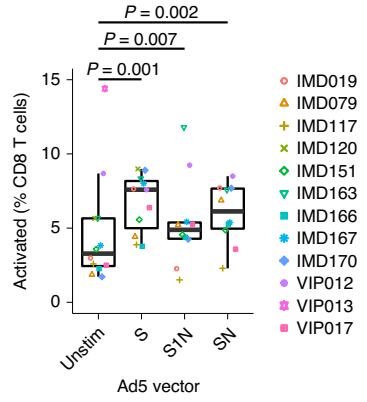

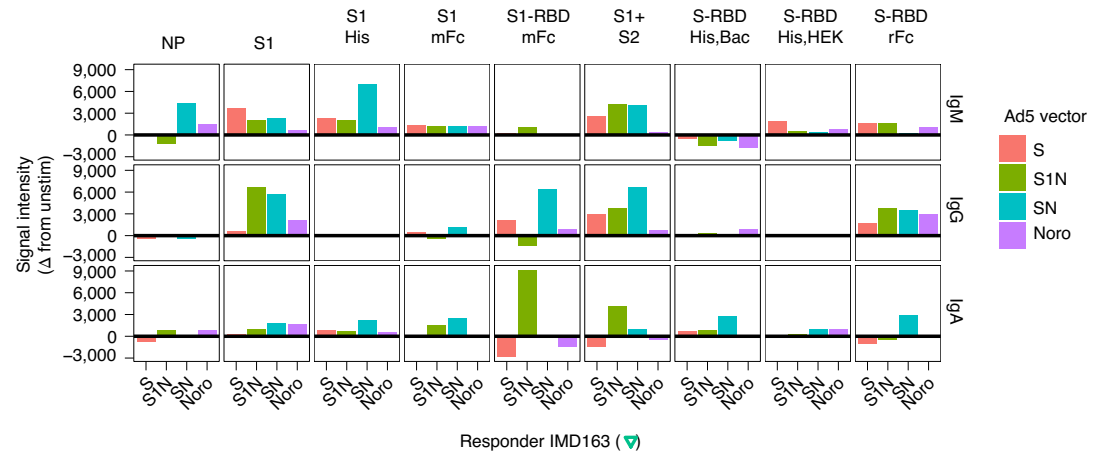

Fig. 6 | Ability to respond to non-influenza antigens and immunomodulation with adjuvants. a,b, Plasmablast differentiation in response to MMR vaccine stimulation in tonsil organoid cultures (a) and their corresponding MMR-specific lgG production (b). Organoid cultures from seven donors were harvested on day 11 for flow cytometry analysis and antibody secretion. Significance values were calculated using paired, two-sided Wilcoxon signed-rank tests. Donor ages are shown in parentheses. c, PE-specific B cells were detected at low levels in unstimulated cultures $(0.05 \%$ of total B cells) and were increased with PE stimulation but not with an irrelevant antigen ( $n=3$ donors; day 11 after stimulation). d, PE-specific $B$ cell frequencies were enhanced by stimulation in the presence of alum ( $n=4$ donors; day 10 after stimulation). Representative flow cytometry plots are shown from one donor. Individual points are shown for each donor. Treatment groups were tested with paired, two-sided $t$-tests to determine significance values. e, Tonsil organoid responses to rabies vaccine, as indicated by pre-GC, GC and plasmablast B cell phenotypes and antigen-specific lgM after $14 \mathrm{~d}$ of culture. NP, nucleoprotein. $P$ values between treatment groups were determined with paired, two-sided $t$-tests $(n=10$ donors). $\mathbf{f}$, Lung-draining lymph nodes and spleen organoids were stimulated for $14 \mathrm{~d}$ with rabies vaccine. $\mathbf{g}$, Tonsil organoids were stimulated with vaccine candidates for SARS-CoV-2 ( $n=12$ donors). Ad5 vectors containing either spike protein (S), spike and nucleocapsid protein (SN) or the S1 spike subunit with nucleocapsid (S1N) were used for stimulation, and responses were measured after $14 \mathrm{~d}$ in culture. Box plots show the median values with hinges representing the first and third quartiles and whiskers representing the highest and lowest value that is within 1.5 times the interquartile range of the hinges. Culture supernatants were collected and tested for antigen-specific IgM, IgG and IgA. One donor that was positive for multiple proteins is shown. An irrelevant adenoviral vector with norovirus VP1 was used as a control. Paired, two-sided Wilcoxon signed-rank tests were used to calculate $P$ values shown (not adjusted for multiple comparisons).

We also characterized the affinities of antibodies against A/California/07/2009 H1N1 HA in $\mathrm{CD}^{+}$-depleted cultures. The dissociation rates for antibodies raised in the absence of $\mathrm{CD}^{+}$ cells were four- to ten-fold higher than wild-type controls (Fig. $5 \mathrm{f}$ and Extended Data Fig. 7c), even when the quantity of specific antibody was comparable between intact and $\mathrm{CD} 4^{+}$-depleted 
cultures. Thus, $\mathrm{CD}^{+} \mathrm{T}$ cells are important for both the magnitude and affinity of the antibody response.

We determined that three to four cell types were minimally required to consistently achieve detectable plasmablast differentiation and naive antibody responses against LAIV: naive B cells, APCs, $\mathrm{CD}^{+} \mathrm{T}$ cells and, in some donors, a mixed population of CD45stromal cells (Fig. 5g). Notably, naive $\mathrm{CD} 4^{+} \mathrm{T}$ cells were either as good or better than memory $\mathrm{CD} 4^{+} \mathrm{T}$ cells in their ability to help $\mathrm{B}$ cells differentiate into plasmablasts and stimulate influenzaspecific antibodies (Fig. 5h).

Response to other antigens and the effect of adjuvants. We next investigated whether tonsil organoids could also respond to non-influenza memory antigens. We stimulated tonsil organoids with the measles, mumps and rubella (MMR) vaccine, which is recommended to be administered at 12 months, and assessed plasmablast formation and antigen-specific IgG responses. These cultures had significantly increased plasmablasts compared to controls (Fig. 6a), and six of the seven donors made measles-specific IgG, while the mumps and rubella responses were weaker and only detected in three of the older children tested (Fig. 6b).

To determine whether tonsil organoids could serve as a platform for priming antigen-specific adaptive responses, we first stimulated cells with naive antigen phycoerythrin (PE), with and without adjuvants. Here we saw a substantial (tenfold) increase in $\mathrm{PE}^{+} \mathrm{B}$ cells in PE-stimulated cultures compared to unstimulated or irrelevant antigen-stimulated cultures (Fig. 6c). The frequency of $\mathrm{PE}^{+} \mathrm{B}$ cells could also be enhanced by alum hydrogel but not imiquimod (Fig. 6d), indicating that at least some naive responses can be elicited and modulated by appropriate adjuvants. We next tested the ability of tonsils, lung-draining lymph nodes and spleen cultures to respond to the $\mathrm{T}$ cell-dependent rabies vaccine. The donors had not previously received this vaccine. Tonsil B cells were more differentiated in vaccine-stimulated cultures after $14 \mathrm{~d}$ in organoid culture and four of ten donors had detectable increases in rabies nucleoprotein-specific IgM (Fig. 6e). Lung-draining lymph node and spleen organoids also showed modest rabies-specific antibody production, with some specific IgM but not IgG production, consistent with a priming response ${ }^{39}$ (Fig. 6f).

Finally, we investigated the ability of tonsil organoids to respond to a series of vaccine candidates developed in response to severe acute respiratory syndrome coronavirus 2 (SARS-CoV-2). These vaccine candidates use a replication-deficient type 5 adenovirus (Ad5) that encodes either the full-length viral spike protein, spike and nucleocapsid protein or the S1 spike subunit with nucleocapsid in the Ad5 E1 region. All tonsil tissue samples were collected before the SARS-CoV-2 pandemic and thus naive to these antigens. On day 14 after stimulation, we observed plasmablast differentiation in a subset of donors and significant CD8 T cell activation compared to unstimulated controls (Fig. 6g). Using a protein microarray to detect antibody specificities against SARS-CoV-2, specific IgG and IgA antibodies were present for some spike and nucleocapsid proteins in a few donors. One particularly robust responder, IMD163, is shown in Fig. $6 \mathrm{~g}$ (data from all donors are available in Extended Data Fig. 8). This shows that immune organoid cultures can be used to analyze and compare vaccine candidates to new and previously encountered antigens.

\section{Discussion}

Although GCs have been studied extensively in mice, to our knowledge this is the first report of an in vitro system supporting antigen-specific somatic hypermutation, affinity maturation and class switching of human B cells. Tonsils are a readily accessible and underutilized source of human immune tissue and contain all the cell types involved in adaptive immunity, including those largely absent from peripheral blood. We used tonsil organoids to characterize features of the human influenza response and validated and extended findings from previous human and murine studies. We show that the $\mathrm{B}$ cell response to influenza vaccine is diverse and can develop from the naive repertoire. We found that preexisting HA-specific $B$ cells had 5-10 heavy-chain nucleotide mutations (2-4\% mutation rate), and LAIV-stimulated organoids prepared with naive-only $B$ cells had 3-9 mutations, which was adequate to make specific antibodies (Fig. 4). These rates are in line with the average $5 \%$ mutation rate measured in influenza-vaccinated adults ${ }^{40}$.

Human immune responses are incredibly variable, as seen in the current SARS-CoV-2 pandemic, and a major goal of vaccine development is to confer protective immunity as broadly as possible. Another useful feature of the organoid system is its ability to assess immune response variability. We found that while the organoids from most donors respond to LAIV, the magnitude and kinetics of those responses vary widely. In a test of 15 donors, organoids prepared from one donor did not respond to LAIV within $7 \mathrm{~d}$ (Fig. 1). This individual was young and not vaccinated against influenza, suggesting that they could be naive to influenza, and organoids prepared from their cells might require more time to mount a response. In our longitudinal analyses (Fig. 2), we identified a nonresponder who was treated with daily inhaled corticosteroids, demonstrating the sensitivity of the system to immunosuppressive agents. Using SARS-CoV-2 vaccine candidates, we found even more variability, with some individuals unable to make B cell and/or $\mathrm{T}$ cell responses. This further reinforces the need for a system like this to assess broad immunogenicity before clinical studies.

Using depletion studies, we established the functional relevance of individual immune cell types to the influenza response. $\mathrm{pDCs}$ were crucial to the antibody response, although they could be replaced by exogenous type I IFN. These data are consistent with a previous study that found that pDCs induce human blood B cell differentiation through type I IFN ${ }^{41}$ but contrast with a murine influenza challenge study that showed pDCs were dispensable to the response ${ }^{42}$, highlighting a difference between murine and human systems. We also identified a role for preexisting plasmablasts/plasma cells in regulating $\mathrm{B}$ cell differentiation (Fig. 5a,b). When plasmablasts were depleted at the initiation of the culture, influenza-specific antibody production was increased compared to wild-type controls. Secondary lymphoid tissues are competitive niches for plasma cell survival outside of the bone marrow ${ }^{43}$, so depletion of preexisting plasma cells may promote antigen-specific plasmablast survival. We demonstrated that $\mathrm{T}$ cell help plays an age-dependent role in the magnitude of the influenza-specific antibody response and showed that $\mathrm{CD} 4^{+} \mathrm{T}$ cells play an additional role in affinity maturation and $\mathrm{B}$ cell selection (Fig. 5). Memory $\mathrm{CD}^{+} \mathrm{T}$ cells from older children better sustained a naive $\mathrm{B}$ cell response, suggesting that $\mathrm{T}$ cell help is refined by in vivo exposure. These findings are in line with an earlier mouse study showing that memory $\mathrm{T}$ cells can contribute to GC responses even though naive B cells typically did not in their system $^{44}$. Stem-specific antibodies were also only detectable when $\mathrm{CD}^{+} \mathrm{T}$ cells were present, suggesting that manipulating $\mathrm{T}$ cell help is a reasonable strategy to consider for future vaccine design.

Previous efforts to create artificial human lymphoid tissues depended on specialized bioreactors ${ }^{45,46}$ or complex in vitro differentiation strategies ${ }^{47}$. Blood-derived mononuclear cells combined with monocyte-derived dendritic cells can stimulate IgM and cytokine secretion consistent with an adaptive immune response $\mathrm{e}^{45,48}$. It is questionable whether in vitro matured dendritic cells recapitulate the conditions that coordinate $\mathrm{T}$ and $\mathrm{B}$ cell responses in lymphoid tissues. More importantly, a major limitation of bioreactor systems is that they do not show mature GCs, class switching or affinity maturation ${ }^{49}$, likely because many cells required for GC function are absent from peripheral blood mononuclear cells. Recent work illustrated that key aspects of the immune microenvironment can be captured in organoids in several studies that focused on conferring immune protection upon transplantation into mice $e^{50,51}$ or 
recapitulating features of the GC response ${ }^{52,53}$. In these studies, designer hydrogel scaffolds for B cells combined with engineered fibroblasts were used to reveal transcriptional feedback loops involved in GC formation ${ }^{52}$ but lacked antigen specificity. Most recently, maleimide-functionalized hydrogel was shown to support GC B cell activation and an antigen-specific response in murine $B$ cell organoid cultures ${ }^{53}$. However, these methods do not incorporate autologous APCs nor T cells that are both crucial to refine the adaptive immune response in vivo. Due to the need for bioengineered fibroblast cell lines to provide necessary survival signals, these techniques are challenging to translate to human cells because of the diversity of human leukocyte antigen genes and generation of mixed leukocyte reactions. Previous techniques to produce in vitro models of human adaptive immunity have not been widely adopted because of reliance on specialized equipment or challenging technical protocols, poor throughput, lack of evidence for antigen-specific responses, affinity maturation and/or absence of cells known to be crucial to important features of adaptive immunity. Although humanized mice are increasingly used, they are expensive to procure and still have many limitations in terms of recapitulating human immunity ${ }^{54}$.

Although this is a powerful system to assess various aspects of adaptive immunity to influenza antigens, there are some limitations. It has been established that the LAIV is less effective than the inactivated formulation in adults ${ }^{55}$, presumably due to the presence of preexisting antibodies at mucosal sites in non-naive individuals. Such conditions could theoretically be mimicked by introducing autologous serum or spiking in influenza-specific antibodies at the initiation of organoid culture. Another challenge is benchmarking against in vivo tissue responses from immunized humans; such studies are now underway to characterize tonsil and lymph node responses to immunization in situ and compare them to organoid cultures. Finally, the system we describe here involves an immunological organ model in isolation. In a true infection or immunization, the antigens first encounter immune cells at a distal site (for example, skin, muscle and nasopharyngeal mucosa) and then migrate to the lymph nodes, tonsils or spleen where GCs form and $B$ cells are selected. Many adjuvants regulate immune cell migration and local microenvironmental characteristics that are not well captured in tonsil organoids in isolation and thus would not be well suited for studying using this model, although adjuvants that act directly on immune cells can be investigated. Future studies will aim to incorporate these migratory features to capture additional features of the adaptive response in humans.

\section{Online content}

Any methods, additional references, Nature Research reporting summaries, source data, extended data, supplementary information, acknowledgements, peer review information; details of author contributions and competing interests; and statements of data and code availability are available at https://doi.org/10.1038/ s41591-020-01145-0.

Received: 12 March 2020; Accepted: 27 October 2020; Published online: 11 January 2021

\section{References}

1. Behring, E. Untersuchungen uber das Zustandekommen der Diphtherie-Immunitat bei Thieren. Dtsch. Med. Wschr 16, 1145-1147 (1890).

2. Behring, E. \& Kitasato, S. Ueber das Zustandekommen der Diphtherie-Immunitat und der Tetanus-Immunitat bei Thieren. Dtsch. Med. Wschr 16, 1113-1114 (1890).

3. Kaufmann, S. H. E. Emil von Behring: translational medicine at the dawn of immunology. Nat. Rev. Immunol. 17, 341-343 (2017).

4. Kaufmann, S. H. Immunology's foundation: the 100-year anniversary of the Nobel Prize to Paul Ehrlich and Elie Metchnikoff. Nat. Immunol. 9, 705-712 (2008).
5. Miller, J. F. The discovery of thymus function and of thymus-derived lymphocytes. Immunol. Rev. 185, 7-14 (2002).

6. Miller, J. F. \& Mitchell, G. F. The thymus and the precursors of antigen reactive cells. Nature 216, 659-663 (1967).

7. Zinkernagel, R. M. \& Doherty, P. C. Restriction of in vitro T cell-mediated cytotoxicity in lymphocytic choriomeningitis within a syngeneic or semiallogeneic system. Nature 248, 701-702 (1974).

8. Allen, C. D., Okada, T. \& Cyster, J. G. Germinal-center organization and cellular dynamics. Immunity 27, 190-202 (2007).

9. De Silva, N. S. \& Klein, U. Dynamics of B cells in germinal centres. Nat. Rev. Immunol. 15, 137-148 (2015).

10. MacLennan, I. C. et al. Extrafollicular antibody responses. Immunol. Rev. 194, 8-18 (2003).

11. Victora, G. D. \& Nussenzweig, M. C. Germinal centers. Annu. Rev. Immunol. 30, 429-457 (2012).

12. Itano, A. A. et al. Distinct dendritic cell populations sequentially present antigen to CD4 T cells and stimulate different aspects of cell-mediated immunity. Immunity 19, 47-57 (2003).

13. Radtke, A. J. et al. Lymph-node resident CD8 $\alpha^{+}$dendritic cells capture antigens from migratory malaria sporozoites and induce $\mathrm{CD}^{+} \mathrm{T}$ cell responses. PLoS Pathog. 11, e1004637 (2015).

14. McCloskey, M. L., Curotto de Lafaille, M. A., Carroll, M. C. \& Erlebacher, A. Acquisition and presentation of follicular dendritic cell-bound antigen by lymph node-resident dendritic cells. J. Exp. Med. 208, 135-148 (2011).

15. Gerner, M. Y., Casey, K. A., Kastenmuller, W. \& Germain, R. N. Dendritic cell and antigen dispersal landscapes regulate T cell immunity. J. Exp. Med. 214, 3105-3122 (2017).

16. Crotty, S. T follicular helper cell differentiation, function, and roles in disease. Immunity 41, 529-542 (2014).

17. Crotty, S. Follicular helper CD4 $\mathrm{T}$ cells $\left(\mathrm{T}_{\mathrm{FH}}\right)$. Annu. Rev. Immunol. 29 , 621-663 (2011)

18. Cyster, J. G. \& Allen, C. D. C. B cell responses: cell interaction dynamics and decisions. Cell 177, 524-540 (2019).

19. Qi, H., Kastenmuller, W. \& Germain, R. N. Spatiotemporal basis of innate and adaptive immunity in secondary lymphoid tissue. Annu. Rev. Cell Dev. Biol. 30, 141-167 (2014).

20. Vinuesa, C. G., Linterman, M. A., Goodnow, C. C. \& Randall, K. L. T cells and follicular dendritic cells in germinal center B cell formation and selection. Immunol. Rev. 237, 72-89 (2010).

21. Jameson, S. C. \& Masopust, D. What is the predictive value of animal models for vaccine efficacy in humans? Reevaluating the potential of mouse models for the human immune system. Cold Spring Harb. Perspect. Biol. 10, a029132 (2018).

22. Buchbinder, S. P. et al. Efficacy assessment of a cell-mediated immunity HIV-1 vaccine (the Step Study): a double-blind, randomised, placebo-controlled, test-of-concept trial. Lancet 372, 1881-1893 (2008).

23. Watkins, D. I., Burton, D. R., Kallas, E. G., Moore, J. P. \& Koff, W. C. Nonhuman primate models and the failure of the Merck HIV-1 vaccine in humans. Nat. Med. 14, 617-621 (2008)

24. Tameris, M. D. et al. Safety and efficacy of MVA85A, a new tuberculosis vaccine, in infants previously vaccinated with BCG: a randomised, placebo-controlled phase 2b trial. Lancet 381, 1021-1028 (2013).

25. James, K., Skibinski, G. \& Hoffman, P. A comparison of the performance in vitro of precision cut tissue slices and suspensions of human spleen with special reference to immunoglobulin and cytokine production. Hum. Antibodies Hybrid. 7, 138-150 (1996).

26. Edwards, K. M., Snyder, P. N., Stephens, D. S. \& Wright, P. F. Human adenoid organ culture: a model to study the interaction of influenza A with human nasopharyngeal mucosa. J. Infect. Dis. 153, 41-47 (1986).

27. Ferro, L. M., Weedon, H. M., Flego, L. R., Beroukas, D. \& Zola, H. An organ fragment culture model to study lymphocyte activation in human lymphoid tissue. Immunobiology 188, 51-61 (1993).

28. Hoffmann, P., Skibinski, G. \& James, K. Organ culture of human lymphoid tissue. I. Characteristics of the system. J. Immunol. Methods 179, 37-49 (1995).

29. Skibinski, G., Hoffmann, P., Radbruch, A. \& James, K. Organ culture of human lymphoid tissue. II. Marked differences in cytokine production and proliferation between slice and suspension cultures of human spleen. J. Immunol. Methods 205, 115-125 (1997).

30. Soto-Rivera, J. et al. Study of HIV-1 transmission across cervical mucosa to tonsil tissue cells using an organ culture. Am. J. Reprod. Immunol. 69, 52-63 (2013).

31. Klinman, N. R. The mechanism of antigenic stimulation of primary and secondary clonal precursor cells. J. Exp. Med. 136, 241-260 (1972).

32. Skibinski, G., Skibinska, A., Deckers, M. \& James, K. Tonsil stromal-cell lines expressing FDC-like properties: isolation, characterization and interaction with B lymphocytes. Dev. Immunol. 6, 273-284 (1998).

33. van Laar, J. M. et al. Sustained secretion of immunoglobulin by long-lived human tonsil plasma cells. Am. J. Pathol. 171, 917-927 (2007). 
34. Grivel, J. C. \& Margolis, L. Use of human tissue explants to study human infectious agents. Nat. Protoc. 4, 256-269 (2009).

35. Jenkinson, E. J. \& Owen, J. J. T cell differentiation in thymus organ cultures. Semin. Immunol. 2, 51-58 (1990).

36. Robinson, J. H. \& Owen, J. J. Generation of T cell function in organ culture of foetal mouse thymus. II. Mixed lymphocyte culture reactivity. Clin. Exp. Immunol. 27, 322-327 (1977).

37. Whittle, J. R. et al. Flow cytometry reveals that $\mathrm{H} 5 \mathrm{~N} 1$ vaccination elicits cross-reactive stem-directed antibodies from multiple Ig heavy-chain lineages. J. Virol. 88, 4047-4057 (2014).

38. Tan, Y. C. et al. High-throughput sequencing of natively paired antibody chains provides evidence for original antigenic sin shaping the antibody response to influenza vaccination. Clin. Immunol. 151, 55-65 (2014).

39. Brinkman, D. M. et al. Vaccination with rabies to study the humoral and cellular immune response to a $\mathrm{T}$ cell dependent neoantigen in man. J. Clin. Immunol. 23, 528-538 (2003).

40. Jackson, K. J. et al. Human responses to influenza vaccination show seroconversion signatures and convergent antibody rearrangements. Cell Host Microbe 16, 105-114 (2014).

41. Jego, G. et al. Plasmacytoid dendritic cells induce plasma cell differentiation through type I interferon and interleukin 6. Immunity 19, 225-234 (2003).

42. Wolf, A. I. et al. Plasmacytoid dendritic cells are dispensable during primary influenza virus infection. J. Immunol. 182, 871-879 (2009).

43. Radbruch, A. et al. Competence and competition: the challenge of becoming a long-lived plasma cell. Nat. Rev. Immunol. 6, 741-750 (2006)

44. Mesin, L. et al. Restricted clonality and limited germinal center reentry characterize memory B cell reactivation by boosting. Cell $\mathbf{1 8 0}$ 92-106 (2020).

45. Giese, C. et al. A human lymph node in vitro-challenges and progress. Artif. Organs 30, 803-808 (2006)
46. Kuzin, I. et al. Long-term immunologically competent human peripheral lymphoid tissue cultures in a 3D bioreactor. Biotechnol. Bioeng. 108, 1430-1440 (2011)

47. Byers, A. M., Tapia, T. M., Sassano, E. R. \& Wittman, V. In vitro antibody response to tetanus in the MIMIC system is a representative measure of vaccine immunogenicity. Biologicals 37, 148-151 (2009).

48. Giese, C. et al. Immunological substance testing on human lymphatic micro-organoids in vitro. J. Biotechnol. 148, 38-45 (2010).

49. Giese, C. \& Marx, U. Human immunity in vitro - solving immunogenicity and more. Adv. Drug Deliv. Rev. 69-70, 103-122 (2014).

50. Purwada, A. et al. Ex vivo engineered immune organoids for controlled germinal center reactions. Biomaterials 63, 24-34 (2015).

51. Purwada, A. \& Singh, A. Immuno-engineered organoids for regulating the kinetics of B cell development and antibody production. Nat. Protoc. 12, 168-182 (2017).

52. Beguelin, W. et al. EZH2 enables germinal centre formation through epigenetic silencing of CDKN1A and an Rb-E2F1 feedback loop. Nat. Commun. 8, 877 (2017).

53. Purwada, A. et al. Ex vivo synthetic immune tissues with $\mathrm{T}$ cell signals for differentiating antigen-specific, high-affinity germinal center B cells. Biomaterials 198, 27-36 (2019).

54. Wagar, L. E., DiFazio, R. M. \& Davis, M. M. Advanced model systems and tools for basic and translational human immunology. Genome Med. 10, 73 (2018).

55. Monto, A. S. et al. Comparative efficacy of inactivated and live attenuated influenza vaccines. N. Engl. J. Med. 361, 1260-1267 (2009).

Publisher's note Springer Nature remains neutral with regard to jurisdictional claims in published maps and institutional affiliations.

(C) The Author(s), under exclusive licence to Springer Nature America, Inc. 2021 


\section{Methods}

Informed consent and sample collection. Whole tonsils from 150 consented individuals undergoing surgery for obstructive sleep apnea, hypertrophy or recurrent tonsillitis were collected in accordance with the Stanford University Institutional Review Board (IRB). Ethics approval was granted by the Stanford University IRB (protocols 30837 and 47690). Written informed consent was obtained from adult participants and from the legal guardians of children aged $0-17$ years; written informed assent was also obtained from children aged 7 years and older. In this cohort, the participants were children aged 2-17 years $(n=57)$ and adults $(n=3)$ who had surgery for obstructive sleep apnea and/or hypertrophy, and overall, tonsil tissue was typically healthy. Whole tonsils were collected in saline after surgery and then immersed in an antimicrobial bath of Ham's F12 medium (Gibco) containing Normocin (InvivoGen), penicillin and streptomycin for $1 \mathrm{~h}$ at $4{ }^{\circ} \mathrm{C}$ for decontamination of the tissue. Tonsils were then briefly rinsed with PBS and processed as needed for culturing (see below).

Donor lung lymph nodes and spleens were provided by the Gift of Hope Organ and Tissue Donor Network to the University of Chicago. These tissues were determined to have IRB Exempt Status by the University of Chicago IRB. Only de-identified demographic information was obtained.

Tissue and organoid preparation. For cryopreservation of tonsil cells, tissue was dissected into roughly $5 \mathrm{~mm} \times 5 \mathrm{~mm} \times 5 \mathrm{~mm}$ pieces and manually disrupted into a suspension by processing through a $100-\mu \mathrm{m}$ strainer with a syringe plunger. Enzymatic dissociation was not necessary and did not improve the response to LAIV from cryopreserved cells. Tissue debris was reduced by Ficoll density gradient separation, although this step was not required for tonsil organoid development. After washing with complete medium (RPMI with glutamax, $10 \%$ FBS, $1 \times$ nonessential amino acids, $1 \times$ sodium pyruvate, $1 \times$ penicillinstreptomycin, $1 \times$ Normocin (InvivoGen) and $1 \times$ insulin/selenium/transferrin cocktail (Gibco)), cells were enumerated and frozen into aliquots in FBS $+10 \%$ DMSO. Frozen cells were stored at $-140^{\circ} \mathrm{C}$ until use.

For lung-draining lymph nodes and spleen samples, tissues were collected in saline or Hank's Balanced Salt Solution, diced into small pieces and pressed through nylon mesh (Nitex) to break up the tissue. Cells were isolated with Ficoll density gradient separation, washed, enumerated and frozen in FBS + 10\% DMSO. Frozen samples were stored in liquid nitrogen until use.

For culture of cryopreserved cells, aliquots were thawed into complete medium, enumerated and resuspended to $6 \times 10^{7}$ cells per $\mathrm{ml}$ for larger cultures or $2 \times 10^{7}$ cells per $\mathrm{ml}$ for smaller cultures. Cells were plated, $100 \mu \mathrm{l}$ per well, into permeable (0.4- $\mu \mathrm{m}$ pore size) membranes (24-well size PTFE or polycarbonate membranes in standard 12-well plates or 96-well polycarbonate membrane plates with single-well receiver trays; Corning or Millipore), with the lower chamber consisting of complete medium ( $1 \mathrm{ml}$ for 12 -well plates, $200 \mu \mathrm{l}$ for 96 -well plates) supplemented with $1 \mu \mathrm{g} \mathrm{ml}^{-1}$ of recombinant human B cell-activating factor (BAFF; BioLegend). Adding a small amount of BAFF improved total B cell survival (and thus increased overall cell recovery) but was not a requirement for plasmablast differentiation or antibody secretion.

LAIV $\left(1 \mu\right.$ per well, equivalent of $1.6 \times 10^{4}$ to $1.6 \times 10^{5}$ fluorescent focus units per strain; FluMist Quadrivalent, Medimmune), wild-type influenza virus (A/California/07/2009 pandemic strain; a gift from H. Greenberg and X.-S. He), MMR vaccine ( $5 \mu$ l per culture; Merck), R-phycoerythrin ( $1 \mu \mathrm{g}$ per culture; Thermo Fisher), rabies vaccine ( $10 \mu \mathrm{l}$ per culture; Imovax, Sanofi Pasteur) or Ad5-vectored SARS-CoV-2 vaccine candidate $\left(1 \times 10^{8}\right.$ infectious units per culture; Vaxart) was then added directly to the cell-containing portion of the culture setup. For adjuvant testing, alum ( $0.01 \%$; InvivoGen, sold as a $2 \%$ stock wet gel with 9-11 $\mathrm{mg} \mathrm{ml}^{-1}$ stock aluminum content) or imiquimod $\left(2.5 \mu \mathrm{g} \mathrm{ml}^{-1}\right.$; InvivoGen) was added directly into the culture immediately after antigen addition. Cultures were incubated at $37^{\circ} \mathrm{C}, 5 \% \mathrm{CO}_{2}$ with humidity and supplemented with additional medium to the lower wells as necessary. Hypoxic conditions $\left(5 \% \mathrm{O}_{2}\right)$ were tested for $\mathrm{B}$ cell differentiation and antibody secretion and were not significantly different from cultures maintained at standard incubator oxygen levels (17-21\%).

For the Ad5-vectored vaccine candidates, recombinant adenoviral constructs were produced using the publicly available SARS-CoV-2 DNA sequence (GenBank accession no. MN908947.3). Spike and nucleocapsid protein sequences were synthesized and codon optimized for expression in human cells and cloned into the E1 region as previously described ${ }^{56}$. The same vector backbone has been used previously in clinical trials for oral recombinant adenovirus tablets ${ }^{57,58}$. The Ad-S adenoviral vector contains a spike protein under the human cytomegalovirus (CMV) promoter. The Ad-SN vector contains spike under the CMV promoter and nucleocapsid under the human beta-actin promoter. The recombinant Ad-S1N vector uses a fusion sequence combining the S1 region of the SARS-CoV-2 spike gene (including the native furin site between S1 and S2) with the full-length SARS-CoV-2 nucleocapsid gene. All vaccine candidates were purified by cesium chloride density centrifugation and provided in a liquid form for cell culture experiments.

Flow cytometry. Organoids were harvested from the upper portion of the permeable membranes by rinsing the membranes with PBS. Cells were washed with FACS buffer (PBS + 0.1\% BSA, $0.05 \%$ sodium azide and $2 \mathrm{mM}$ EDTA) and stained at $4{ }^{\circ} \mathrm{C}$ with the following anti-human antibodies in the presence of $\mathrm{Fc}$ block and live/dead Aqua Zombie stain, all from BioLegend unless otherwise noted: FITC CD138 (1/100), FITC CD116 (1/100), FITC CD21 (1/50), FITC or Ax488 CXCR5 (1/33), PerCP-Cy5.5 CD8 (1/100), PerCP-Cy5.5 CD33 (1/100), PE CD19 (1/100), PE CD56 (1/100), PE gamma-delta TCR (1/100), PE-Cy7 CD27 (1/100), PE-Cy7 CD123 (1/50), PE-Cy7 CD8 (1/100), APC CD38 (1/200), APC HLA-DR (1/100), APC CD27 (1/200), Ax700 CD45 (1/100), Ax700 CD14 (1/100), APC-Cy7 IgD (1/50), APC-Cy7 CD16 (1/100), APC-Cy7 CD45RA (1/100), Pacific Blue HLA-DR (1/100), Pacific Blue PD-1 (1/50), BV605 CD3 (1/100), BV650 CD4 (1/100), BV650 CD19 (1/100), BUV395 IgM (1/20; BD Biosciences) and BUV395 CD45RA (1/20; BD Biosciences).

For AID staining, after surface staining, the cells were fixed and permeabilized (eBioscience) and stained intracellularly with biotinylated anti-AID antibody (1/100; clone mAID-2; eBioscience) followed by PE-streptavidin (eBioscience). A no-AID antibody control was used to discriminate positive signal. All analyzer data were collected on BD LSRII instruments and analyzed using FlowJo (TreeStar).

Tetramer staining. HLA-A2 tetramers were prepared as previously described ${ }^{59}$, with ultraviolet (UV)-sensitive peptide cleavage and exchange for the A2 immunodominant influenza M1 peptide (GILGFVFTL) or an irrelevant CMV pp65 peptide (NLVPMVATV) as a control; tetramers were prepared from the peptide-exchanged monomers by conjugation to PE-streptavidin and APC-streptavidin, respectively (eBioscience). For staining, HLA-A2 donor organoids were harvested, washed with FACS buffer and stained for $1 \mathrm{~h}$ at $4{ }^{\circ} \mathrm{C}$ with tetramers $(0.5 \mu \mathrm{g}$ of monomer per test) in the presence of Fc block. During the last $30 \mathrm{~min}$ of tetramer staining, lineage-defining antibodies were added, and samples were washed with FACS buffer. For analysis, influenza M1 tetramer-positive CD8 ${ }^{+}$ $\mathrm{T}$ cells were identified as single-positive $\mathrm{T}$ cells for the influenza tetramer (negative for CMV tetramer) and with staining above a no-influenza-tetramer staining control.

Antibody detection by ELISA. For detection of influenza-specific antibodies, ELISA plates (Costar) were coated with $0.1 \mu \mathrm{g}$ per well of season-matched Fluzone Quadrivalent inactivated influenza vaccine (based on reported total HA content from the manufacturer; Sanofi) to act as the capture antigen. For A/California HA antibody detection, recombinantly expressed soluble HA trimers were used in place of the inactivated vaccine as the capture antigen. Diluted (1:20 or 1:50) culture supernatants were added to coated, blocked plates. A human pan-influenza monoclonal IgG antibody (H1N13-M; Alpha Diagnostics) was used as a standard to estimate specific antibody concentration for experiments where antibody concentration is quantitatively defined. Horseradish peroxidase-conjugated anti-human secondary antibodies to either IgM/IgG/IgA (Sigma) or Fc-IgG alone (Bethyl; adsorbed for other isotypes) were used to detect bound antibodies. Plates were developed with TMB substrate solution (Thermo Scientific), quenched with sulfuric acid and read at $450 \mathrm{~nm}$. Neutralization experiments were performed by Monogram Biosciences with a pseudovirus neutralization assay with HA matching the vaccine antigen strains.

For detection of total IgG, culture supernatants were diluted at 1:500 and assayed by ELISA (Thermo Scientific) following manufacturer's instructions. For detection of MMR-specific IgG, culture supernatants were tested by ELISA (Abcam) following the manufacturer's instructions. Supernatants were diluted at 1:2.5 with the provided sample diluent and serial twofold dilutions out to 1:40 confirmed signal specificity (data not shown). To measure rabies nucleoprotein-specific IgM and IgG, supernatants were diluted at 1:5 with specific IgM and IgG detection kits (Alpha Diagnostic).

Antibody detection by protein microarray. Organoid cultures stimulated with recombinant adenoviral vectors with SARS-CoV-2 sequences were tested for specific antibody production using a protein microarray. The technology has been previously described for the detection of influenza-specific antibodies ${ }^{60}$ and was recently adapted to include commercially available SARS-CoV-2 proteins $^{61}$. The SARS-CoV-2 proteins are commercially available from Sino Biological. Briefly, day-14 culture supernatants were diluted 1:1 with blocking buffer and incubated for $30 \mathrm{~min}$. Then, diluted samples were added to the microarray for overnight hybridization. The array was washed three times with Tris-buffered saline containing Tween20, then treated with Qdot-conjugated anti-IgM, IgG or IgA secondary antibodies for $2 \mathrm{~h}$. After another three washes, the array was dried and read, with signal intensities representing relative antibody quantities bound to each protein spot.

Immunofluorescence. Immunofluorescence microscopy samples were prepared from frozen tonsil cells stimulated with LAIV and harvested 4 or $7 \mathrm{~d}$ after stimulation. Permeable membrane inserts containing organoids were gently immersed in PBS, fixed with $4 \%$ paraformaldehyde in PBS for $30 \mathrm{~min}$ at $4{ }^{\circ} \mathrm{C}$ and washed three times with water. Cultures were kept at room temperature and incubated for $20 \mathrm{~min}$ in increasing concentrations of warmed $\left(37^{\circ} \mathrm{C}\right) \mathrm{OCT}$ Compound (Fisher) diluted in PBS at $25 \%, 50 \%$ and $75 \%$ (vol/vol), with a final incubation in pure OCT. Samples were snap frozen on dry ice, inserts were removed with forceps, and samples were embedded in an additional layer of OCT and frozen. 
Embedded samples were sectioned at $25 \mu \mathrm{m}$ and adhered to poly-L-lysine-coated coverslips. Sections were dried for $4 \mathrm{~min}$ in a dehumidified chamber and permeabilized in acetone for $10 \mathrm{~min}$ at room temperature. Rehydration was performed in stain buffer ( $1 \%$ BSA, $1 \%$ normal goat serum and $0.01 \%$ sodium azide in PBS) for $30 \mathrm{~min}$. Sections were stained first with primary unconjugated antibodies at room temperature for $3 \mathrm{~h}$. Secondary antibody staining was performed for $1 \mathrm{~h}$ at room temperature followed by primary direct conjugate antibodies for $3 \mathrm{~h}$ at room temperature.

Primary antibodies. Immunofluorescence staining was performed using the following antibodies at the stated concentrations: BioLegend: CD3-BV421 (SK7; 1:25), CD4-AF594 (RPA-T4; 1:50), CD19-AF647 (SJ25C1; 1:25), CXCR4 (12G5; 1:100), CD21-FITC (Bu32; 1:100), PD-1-AF488 (NAT105; 1:100), BCL-6 (IG191E/ A8; 1:50); Sigma-Aldrich: CXCR5 (Polyclonal; 1:100), CD83 (Polyclonal; 1:100); Thermo Fisher: AID (ZA001; 1:100), CD8-AF488 (AMC908; 1:100), CD20-eFluor 615 (L26; 1:100), CD20-eFluor 660 (L26; 1:100); BD Biosciences: Ki67-BV421 (B56; 1:5); Fisher Science: IgD-AF488 (IgD26; 1:10).

Secondary antibodies. The following secondary antibodies from Thermo Fisher and their dilutions were used: goat anti-mouse $\operatorname{IgG}(\mathrm{H}+\mathrm{L})$ AF Plus 555 (1:200), goat anti-rabbit IgG $(\mathrm{H}+\mathrm{L})$ AF Plus 555 (1:200), goat anti-rabbit IgG $(\mathrm{H}+\mathrm{L})$ AF Plus 594 (1:200).

After collecting imaging data from antibody markers, slides were stained with $1 \mu \mathrm{g} \mathrm{ml}^{-1} \mathrm{DAPI}$ to visualize nuclear staining. Imaging was performed on an Inverted Zeiss LSM 880 confocal instrument using $\times 25$ magnification. Sample $z$-stacks were taken at $2-\mu \mathrm{m}$ slices. DAPI was stimulated at $405 \mathrm{~nm}$, FITC/Alexa Fluor 488 at $488 \mathrm{~nm}$, Alexa Fluor 555 at $561 \mathrm{~nm}$ and Alexa Fluor 594 at $594 \mathrm{~nm}$. Images were processed in ImageJ (version 2.0.0). Tile stitching was performed with the Grid/Collection stitching tool using positions from files in the order defined by the image metadata. The fusion method used was Linear Blending, with Computer Overlap and Ignore Calibration (all other parameters were set to default). After despeckling, $z$-stacks were merged using ZProjection by Maximum Intensity and contrast adjusted to better present structure, and channels were stacked to RGB and a scale bar added.

To quantify the fraction of CXCR4- and CD83-expressing cells in GC areas, GCs were cropped into two portions that resembled light and dark zone-like regions as shown on the imaging figure. CD20-, CD83- and CXCR4-positive cells were manually counted (multipoint tool in ImageJ) in addition to double- and triple-positive cells. CD $83^{+} \mathrm{CXCR} 4^{-} \mathrm{CD} 20^{+}$and $\mathrm{CD} 83^{-} \mathrm{CXCR} 4^{+} \mathrm{CD} 20^{+}$cells in each region were calculated and presented as a proportion of the total $\mathrm{CD} 20^{+}$population from the same area. To determine the mean intensities of marker expressions in light and dark zone regions, images were converted to RGB using 'stack to RGB' and then 'color histogram' was performed on each channel using ImageJ, which displays a histogram of the intensity values. The mean of each channel was used to compare the average marker expression between different regions of equivalent size.

ELISpot. ASCs were detected using an ELISpot protocol based on work by Shah and Koelsch ${ }^{62}$. Briefly, cultures that were either stimulated for $7 \mathrm{~d}$ with LAIV or left unstimulated were resuspended and enumerated, then plated on inactivated influenza vaccine-coated and blocked 96-well PVDF membrane plates (Millipore). Each sample was plated with three, threefold dilutions in triplicate, and total live-cell counts ranged from $2.22 \times 10^{4}$ to $1.07 \times 10^{5}$ cells per well at a $1: 9$ dilution, which was used for enumeration of ASCs. Cells were incubated on these membranes, undisturbed for $5 \mathrm{~h}$ at $37^{\circ} \mathrm{C}$. Plates were then washed and treated with horseradish peroxidase-conjugated anti-IgG/IgA/IgM secondary antibody. After incubation overnight at $4^{\circ} \mathrm{C}$, plates were washed and developed with AEC substrate (BD), washed 20 times with water, dried, and spots were enumerated. The frequency of ASCs out of total B cells was determined from B cell flow cytometry data analysis and the direct cell enumeration counts.

Single-cell RNA-seq. Cells from either day-0 tonsils (processed, cryopreserved and thawed) or organoids (from day 5 and day 9 after stimulation with LAIV or left unstimulated) were stained with a mixture of fluorophore-conjugated antibodies to enable sorting for $\mathrm{CD} 45^{+} \mathrm{CD} 19^{+} \mathrm{CD}^{-} \mathrm{B}$ cells and for sequencing detection of DNA-tagged antibodies (CD20 clone L27, CD19 clone SJ25C1, CD71 clone L01.1, IgM clone G20-127, IgA clone A59, CD161 clone HP-3G10, CD27 clone L128, CD38 clone HIT2, IgD clone IA6-2, IgG clone G18-145, CD83 clone HB15E, CXCR4 clone 12G5, TCR Vg9 clone B3, CD3 UCHT1, CD4 clone SK3, CD8 clone RPA-T8 and CXCR5 clone RF8B2). A cocktail of DNA-tagged antibodies was used to allow manual gating on $\mathrm{B}$ cells $\left(\mathrm{CD} 19^{+} \mathrm{CD}^{-}\right)$and $\mathrm{B}$ cell subsets $(\mathrm{CD} 27$ and CD38) to identify GC B cells and naive B cells. The sorted cells were tagged with sample barcodes to enable pooling. The cells were loaded and captured from the pooled sample using the BD Rhapsody pipeline following the manufacturer's instructions to prepare the libraries, using the targeted human immune gene panel for amplification. Libraries were sequenced using Illumina Novaseq platforms and the resulting data processed using the Rhapsody analysis pipeline. Using SeqGeq (BD) software, individual samples were debarcoded, and B cell subsets were gated based on DNA-barcoded antibodies for CD3, CD19, CD27 and CD38. Individual populations were then exported along with their gene expression profiles for analyses. UMAP dimensionality reduction was run using the R package 'umap' on gene (and not DNA-tagged antibody) expression profiles. For fold change expression analyses, genes that were overexpressed in GC B cells by at least 1.5 -fold were plotted.

B cell receptor sequencing. For isotype-switching analysis, tonsil cells from day- 0 or day-7 organoids were harvested, washed with FACS buffer, and stained with a cocktail of lineage-defining antibodies as above in the presence of Fc block, then bulk sorted using a FACS Fusion or Aria II instrument. Bulk sequencing of immunoglobulin heavy-chain gene rearrangements for isotype-switching analysis was carried out as previously reported ${ }^{40,63}$. Briefly, RNA was isolated from sorted (memory CD $38^{-} \mathrm{CD} 27^{+}$, GC CD $38^{+} \mathrm{CD} 27^{+}$and plasmablast $\mathrm{CD} 38^{+++} \mathrm{CD} 27^{+}$) cells using Trizol (Thermo Fisher) and reverse transcribed to cDNA using Superscript II (Life Technologies) primed with random hexamer primers. Amplicons from IgM, IgD, IgG, IgA and IgE were PCR amplified in separate reactions, using IGHV framework region 1 primers and isotype primers in the first constant region exon, modified to contain partial Illumina linker sequences, sample barcode sequences and randomized nucleotides to ensure sequence diversity in the initial cycles of sequencing (as described by Roskin et al. ${ }^{64}$ ). A second PCR was carried out to complete the Illumina linker sequences before amplicon quantification, pooling, gel extraction (Qiagen) and sequencing on an Illumina MiSeq instrument with 600 -cycle kits as $2 \times 300$ paired-end reads ${ }^{40,63}$. Bulk BCR heavy-chain sequences were analyzed with an in-house developed pipeline, based on IgBLAST for V, D and J gene segment alignment and CDR-H3 parsing ${ }^{40,63}$. Clonally related sequences in the bulk sequencing data were identified based on shared usage of IGHV and IGHJ genes, equal CDR-H3 length and single-linkage clustering of CDR-H3 nucleotide sequences at a $90 \%$ identity threshold.

For analyses of somatic hypermutation and A/California 2009 HA-specific B cells, tonsil cells from day- 0 or day-7 organoids were harvested, washed with FACS buffer, then treated with $2 \mu \mathrm{g}$ per sample $\left(4 \mu \mathrm{g} \mathrm{ml}^{-1}\right)$ of biotinylated recombinant A/California influenza HA1 hemagglutinin (Y98F mutant; a gift from B. Graham and the Vaccine Research Center) in the presence of Fc block, then washed and stained with $0.2 \mu \mathrm{g} \mathrm{ml}^{-1}$ of fluorescently labeled streptavidin and a cocktail of lineage-defining antibodies.

For single-cell sorting, $\mathrm{HA}^{+} \mathrm{B}$ cells of $\mathrm{GC}$ or plasmablast phenotype $\left(\mathrm{CD} 38^{+} \mathrm{CD} 27^{+}\right.$or $\mathrm{CD} 38^{+++} \mathrm{CD} 27^{+}$, respectively) were sorted. Single-cell antibody sequencing was performed as previously described ${ }^{38,65-67}$. After single-cell sorting into 96-well plates, cDNAs were labeled with well-specific barcode oligonucleotides and pooled by plate, followed by gene-specific PCR and library preparation with previously reported primer sequences ${ }^{65}$. Libraries were sequenced with Illumina MiSeq $2 \times 300$ paired-end sequencing. Sequence analysis was performed as previously described ${ }^{65}$. Briefly, fastq generation and plate demultiplexing were completed using the onboard MiSeq Generate FASTQ workflow. After quality filtering, paired reads were stitched, separated by well ID and consensus sequences determined by clustering well ID reads into operational taxonomic units ${ }^{68}$. Consensus operational taxonomic unit sequences were analyzed with version 1.5.7.1 of IMGT HighV-QUEST ${ }^{69}$. For single-cell data, clonal families were defined by the same $\mathrm{V}$ and J gene usage and at least $70 \%$ amino acid identity in the CDR3 locus for both heavy and light chains. A caveat of single-cell HA-specific B cell sequencing is that the most vigorously responding $B$ cells may have reduced surface immunoglobulin expression as they convert to antibody secretion, and so it is possible that the highest ASCs could not be captured during HA-specific B cell sorting.

Bulk IgH sequencing and analysis was performed using MIDCIRS as previously described ${ }^{70}$. Sequencing was performed on an Illumina MiSeq with the v3, 600-cycle kit. mRNA molecules were tagged with $12 \mathrm{~N}$ randomized molecular identifiers (MIDs) during reverse transcription. Reads with the same MID were grouped together and then further clustered into subgroups based on $85 \%$ sequence similarity to separate distinct mRNA molecules that were tagged with the same MID. Consensus sequences were then formed from the MID subgroups to average out PCR and sequencing errors and mitigate amplification and sequencing bias. Clonal lineages were defined using single-linkage clustering on the consensus sequences with the same criteria as above. The size of the clonal lineage refers to the total number of consensus sequences, or mRNA molecules, that make up the lineage, while the diversity of the lineage refers to the number of unique consensus sequences within the lineage.

To infer HA specificity of total, unsorted B cells from day-7 tonsil cultures, all FACS-sorted, HA-specific B cell sequences were first grouped together $(n=20,977)$ Then, all nucleotide sequences were translated to amino acid sequences, and each day-7 sequence was aligned to the pool of $\mathrm{HA}^{+}$sequences to find the minimum distance to the nearest HA-specific sequence. If any sequence within a clonal lineage was found to be an exact match or one substitution away from a known $\mathrm{HA}$-specific sequence, the lineage was labeled 'HA-inferred'. Clonal lineages were then split into HA-inferred and non-A/California HA for further analysis.

Affinity binding experiments. Binding affinities of the indicated antigens to antibody-containing supernatant samples were determined by biolayer interferometry using an Octet QK instrument (Pall ForteBio). For analysis of the 
effects of T cells on antibody responses, antibodies were purified from culture supernatants using a Protein G affinity column (GE Healthcare Life Sciences). The full-length, head and stem domain $\mathrm{H} 1 \mathrm{CA} / 09 \mathrm{HA}$ antigens were purified as described previously using a Ni-NTA affinity column followed by size-exclusion cleanup $^{71,72}$. The purified antigens were captured on anti-penta-his (HIS1K) biosensor tips in PBS-T (PBS with $0.05 \%$ Tween 20 (pH 7.4)). The ligand-bound sensors were dipped into control wells or purified antibodies $(200-500 \mathrm{nM})$. A similar antibody concentration was used for all the evaluated conditions from an individual donor. Unliganded sensors dipped into the analyte served as controls for nonspecific binding. The traces were processed using ForteBio Data Analysis Software (v8.0). The data were fitted globally to a simple 1:1 Langmuir interaction model to obtain the kinetic parameters. Each binding interaction was repeated at least thrice.

Cell depletion experiments. Thawed tonsil cells were stained and bulk sorted into culture medium using a FACS Aria II or Fusion (BD). Sorting experiments involved separating individual cell subsets using the following markers: myeloid

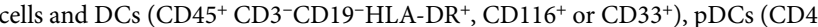
$\left.5^{+} \mathrm{CD} 3^{-} \mathrm{CD} 19^{-} \mathrm{HLA}-\mathrm{DR}{ }^{+} \mathrm{CD} 123^{+}\right), \mathrm{T}_{\text {reg }}$ cells $\left(\mathrm{CD} 45^{+} \mathrm{CD} 3^{+} \mathrm{CD} 19^{-} \mathrm{CD} 4^{+} \mathrm{CD} 25^{+}\right.$ $\left.\mathrm{CD} 127^{\mathrm{lo}}\right)$, total B cells $\left(\mathrm{CD} 45^{+} \mathrm{CD} 3^{-} \mathrm{CD} 19^{+}\right)$, non-naive $\mathrm{B}$ cells $\left(\mathrm{CD} 38^{+}\right.$and/or $\mathrm{CD}^{2} 7^{+} \mathrm{IgM}^{-}$and/or $\left.\mathrm{IgD}^{-}\right)$, naive B cells $\left(\mathrm{CD} 38^{-} \mathrm{CD} 27^{-} \mathrm{IgM}^{+} \mathrm{IgD}^{+}\right)$, pre-GC B cells $\left(\mathrm{CD} 38^{+} \mathrm{CD} 27^{-}\right)$, GC B cells $\left(\mathrm{CD} 38^{+} \mathrm{CD} 27^{+}\right)$, memory B cells $\left(\mathrm{CD} 38^{-} \mathrm{CD} 27^{+}\right)$and plasmablasts/plasma cells $\left(\mathrm{CD} 38^{+++} \mathrm{CD} 27^{+}\right)$. Cell types were depleted by FACS from day- 0 tonsil cells and cultured with LAIV for $7 \mathrm{~d}$. As controls, depleted cultures were reconstituted with the cell type that was originally sorted and plated at the same cell density as the depleted cultures. For depletion of HA-specific B cells, tonsil samples were stained as described in 'BCR sequencing' and HA-specific $\mathrm{B}$ cells were defined as $\mathrm{CD} 45^{+} \mathrm{CD} 19^{+} \mathrm{CD}^{-} \mathrm{HA}^{+}$. A post-sort analysis was used to ensure depletion purity.

Statistical analysis. All statistical analyses were performed in R. For comparing paired samples (frequencies of plasmablasts and antibody secretion in unstimulated versus LAIV-stimulated cultures, standard versus Transwell responses, unstimulated versus MMR-stimulated cultures, somatic hypermutation analyses, studying the effect of $\mathrm{CD} 4^{+}$cell depletion on plasmablast differentiation and specific antibody secretion), paired Wilcoxon signed-rank tests (two-sided) were performed. To analyze the CD4 depletion effect in young versus older children, unpaired Mann-Whitney U tests (two-sided) were performed. Welch's $t$-tests (two-sided) were used to compare the somatic hypermutation levels between culture conditions and the clone size and diversity of inferred HA-specific and nonspecific B cell lineages. Two-sided, paired $t$-tests were used to compare the effect of PE stimulation with and without adjuvants on the frequency of $\mathrm{PE}^{+} \mathrm{B}$ cells.

Reporting Summary. Further information on research design is available in the Nature Research Reporting Summary linked to this article.

\section{Data availability}

Requests for raw data, analyzed data and materials will be reviewed by Stanford University to determine if the request is subject to intellectual property or confidentiality obligations. Data and materials that can be shared will be released using a Material Transfer Agreement. Targeted single-cell RNA-seq data are available in the Gene Expression Omnibus under GSE158459.

\section{References}

56. He, T. C. et al. A simplified system for generating recombinant adenoviruses. Proc. Natl Acad. Sci. USA 95, 2509-2514 (1998).

57. Kim, L. et al. Safety and immunogenicity of an oral tablet norovirus vaccine, a phase I randomized, placebo-controlled trial. JCI Insight 3, e121077 (2018)

58. Liebowitz, D. et al. Efficacy, immunogenicity and safety of an oral influenza vaccine: a placebo-controlled and active-controlled phase 2 human challenge study. Lancet Infect. Dis. 20, 435-444 (2020).

59. Toebes, M. et al. Design and use of conditional MHC class I ligands. Nat. Med. 12, 246-251 (2006).

60. Nakajima, R. et al. Protein microarray analysis of the specificity and cross-reactivity of influenza virus hemagglutinin-specific antibodies. mSphere 3, e00592-18 (2018).

61. de Assis, R. R., et al. Analysis of SARS-CoV-2 antibodies in COVID-19 convalescent plasma using a coronavirus antigen microarray. Preprint at bioRxiv https://doi.org/10.1101/2020.04.15.043364 (2020).

62. Shah, H. B. \& Koelsch, K. A. B cell ELISpot: for the identification of antigen-specific antibody-secreting cells. Methods Mol. Biol. 1312, 419-426 (2015).
63. Ellebedy, A. H. et al. Defining antigen-specific plasmablast and memory B cell subsets in human blood after viral infection or vaccination. Nat. Immunol. 17, 1226-1234 (2016).

64. Roskin, K. M. et al. IgH sequences in common variable immune deficiency reveal altered B cell development and selection. Sci. Transl. Med. 7, 302ra135 (2015).

65. Blum, L. K. et al. Circulating plasmablasts are elevated and produce pathogenic anti-endothelial cell autoantibodies in idiopathic pulmonary arterial hypertension. Eur. J. Immunol. 48, 874-884 (2018).

66. Kinslow, J. D. et al. Elevated IgA plasmablast levels in subjects at risk of developing rheumatoid arthritis. Arthritis Rheumatol. 68, 2372-2383 (2016).

67. Nair, N. et al. VP4- and VP7-specific antibodies mediate heterotypic immunity to rotavirus in humans. Sci. Transl. Med. 9, eaam5434 (2017).

68. Edgar, R. C. UPARSE: highly accurate OTU sequences from microbial amplicon reads. Nat. Methods 10, 996-998 (2013).

69. Alamyar, E., Duroux, P., Lefranc, M. P. \& Giudicelli, V. IMGT tools for the nucleotide analysis of immunoglobulin and T cell receptor V-(D)-J repertoires, polymorphisms and IG mutations: IMGT/V-QUEST and IMGT/ HighV-QUEST for NGS. Methods Mol. Biol. 882, 569-604 (2012).

70. Wendel, B. S. et al. Accurate immune repertoire sequencing reveals malaria infection driven antibody lineage diversification in young children. Nat. Commun. 8, 531 (2017).

71. Joyce, M. G. et al. Vaccine-induced antibodies that neutralize group 1 and group 2 influenza A viruses. Cell 166, 609-623 (2016).

72. Mallajosyula, V. V. et al. Influenza hemagglutinin stem-fragment immunogen elicits broadly neutralizing antibodies and confers heterologous protection. Proc. Natl Acad. Sci. USA 111, E2514-E2523 (2014).

\section{Acknowledgements}

The authors thank the participants for donating their tissues for this study; J. Cyster, J. Idoyaga and B. Pulendran for thoughtful comments on the manuscript; M. Leipold, C. Tato and R. DiFazio for insightful discussions and troubleshooting suggestions; A. Messner, C. Lopez Angel, R. Cadman, A. Nguyen and H. Song for their assistance with collecting tonsillectomy samples; the Gift of Hope Organ and Tissue Donor Network and J. Solway for tissue procurement through the Gift of Hope; Mike Laine for materials development advice; Mary Rieck for sorting assistance; X. Ji, M. Miranda, I. Goncharov and H. Maecker for single-cell RNA-seq help; and the Cell Sciences Imaging Facility and the Stanford Shared FACS Facility for instrument access. This work was supported by a Bill and Melinda Gates Foundation pilot grant (OPP1113682; to M.M.D.), the National Institutes of Health (NIH) grants (5U19AI05722915 to M.M.D.; 1R01AI127877 and 1R01AI130398 to S.D.B.; U54CA224081, U19AI116484, U01CA217851 and U01DK085527 to C.J.K.) and the Howard Hughes Medical Institute (to M.M.D.). Sorting and analyzing were performed in the Stanford Shared FACS facility on instruments supported by the NIH S10 Shared Instrument Grants (S10RR025518-01 and S10RR027431-01) and the Parker Institute for Cancer Immunotherapy.

\section{Author contributions}

L.E.W., A.S., C.J.K. and M.M.D. conceived the study and guided it throughout; L.E.W., A.S., C.M.C., B.S.W., M.M.L., V.M., L.P.J., J.Z.A., L.K.B., N.G., F.Y., K.J.L.J., K.R. and K.M.R. performed experiments and/or analyzed and interpreted data; L.E.W., A.I.S., K.M.B., K.D.M., I.N.A., G.B.H., P.S.K., W.H.R., S.D.B., C.J.K. and M.M.D. contributed to sample acquisition efforts, assay development or conceptual design of the study; M.M.L., M.C., E.G.D. and S.N.T. developed and produced antigens; L.E.W. and M.M.D. wrote the paper, and all authors reviewed and approved the manuscript.

\section{Competing interests}

S.N.T., M.C. and E.G.D. are employed and have stock options with the biotechnology company Vaxart. P.L.F. and D.H.D. have shares in Nanommune, a company that uses Sino Biological's proteins on commercially available protein microarrays. L.E.W., A.S., C.M.C., B.S.W., M.M.L., V.M., L.P.J., J.Z.A., L.K.B., N.G., K.J.L.J., F.Y., K.R., K.M.R., K.M.B., K.D.M., I.N.A., A.I.S., A.J., G.B.H., P.S.K., W.H.R., S.D.B., C.J.K. and M.M.D. declare no competing interests.

\section{Additional information}

Extended data is available for this paper at https://doi.org/10.1038/s41591-020-01145-0.

Supplementary information is available for this paper at https://doi.org/10.1038/ s41591-020-01145-0.

Correspondence and requests for materials should be addressed to M.M.D.

Peer review information Saheli Sadanand was the primary editor on this article and managed its editorial process and peer review in collaboration with the rest of the editorial team.

Reprints and permissions information is available at www.nature.com/reprints. 

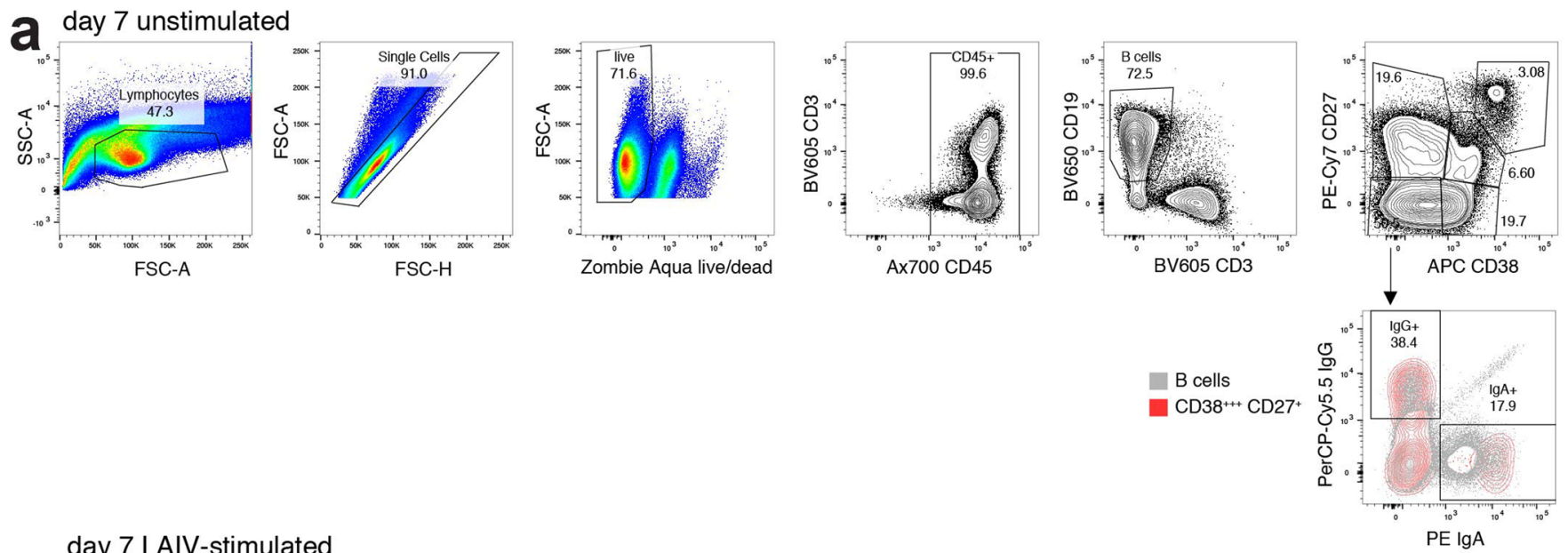

day 7 LAIV-stimulated

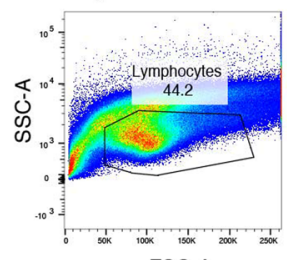

FSC-A

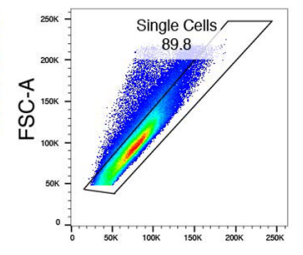

FSC-H

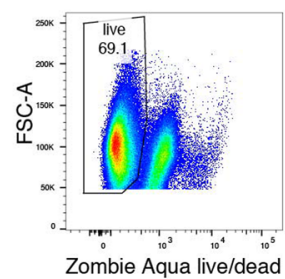

Zombie Aqua live/dead b

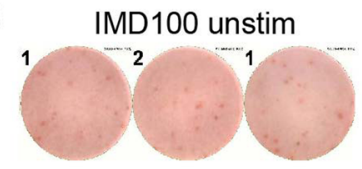

$4.07 \times 10^{4}$ cells/well $60.95 \%$ B cells

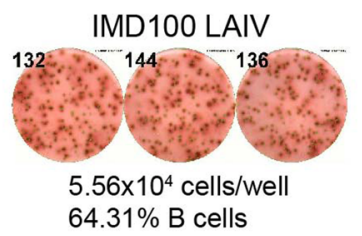

$p=0.001$
C

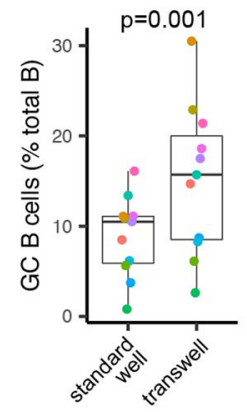

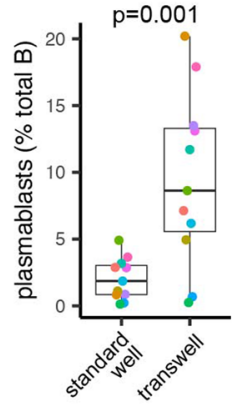

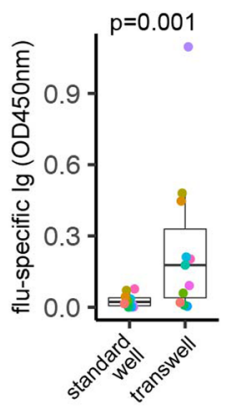

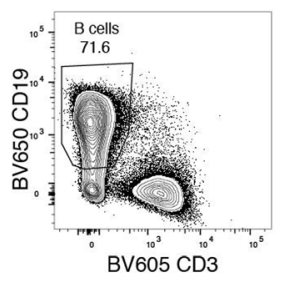
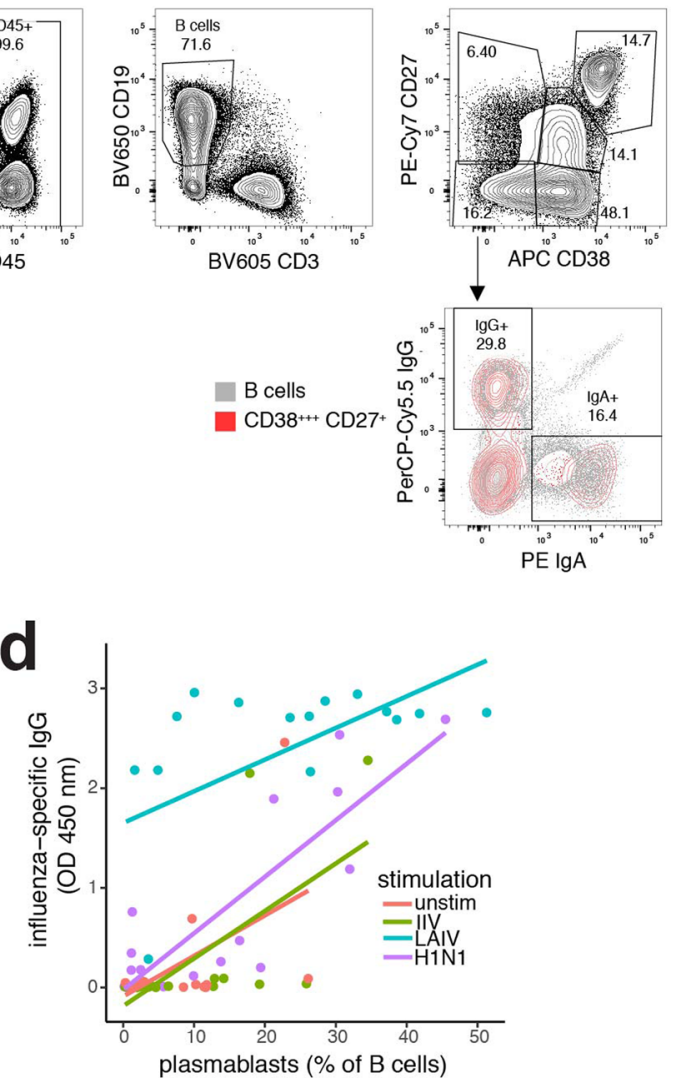

Extended Data Fig. 1 | Characteristics of B cells from tonsil organoid cultures. a, Flow cytometry gating scheme on representative unstimulated and LAIV-stimulated cultures. b, Representative ELISpot wells for detection of Ab-secreting cells with influenza vaccine specificity. The number of spots detected are shown in the corner of each well. c, Comparison of organoid cultures grown in standard flat-bottom wells vs. transwells. Data shown are from day 7 LAIV-stimulated cultures ( $n=6$ donors). p values shown were determined with paired Wilcoxon signed-rank two-sided tests. Boxplots show median values with hinges representing first and third quartiles and whiskers representing the highest and lowest value that is within $1.5 \mathrm{X}$ the interquartile range of the hinges. $\mathbf{d}$, Correlation between specific Ab secretion and plasmablast frequency in influenza-stimulated and unstimulated cultures on day 7. Detection of influenza-specific $\lg G$ antibodies by semi-quantitative indirect ELISA. Optical density was corrected for background using unspent culture medium as a control. Each donor $(n=15)$ was tested under four conditions: no stimulation, IIV-stimulated, LAIV-stimulated, or H1N1 WT virus-stimulated. Plasmablast $\left(\mathrm{CD} 19^{+} \mathrm{CD} 8^{+++} \mathrm{CD} 27^{+}\right)$frequencies were determined by flow cytometry. 



Extended Data Fig. 2 | See next page for caption. 
Extended Data Fig. 2 | Confocal imaging showing distribution of different cell types and their interactions in tonsil tissue and organoid cultures. Merge does not include DAPI staining for clarity. a, One fresh tonsil tissue was stained with panels of markers to define $T$ and B cell areas and GC structure. b, Day 4 unstimulated organoid stained for T and B cell markers. c, Day 4 LAIV organoid stained for germinal center markers. d, Day 4 unstimulated organoid stained for B and T cell distribution. e, Day 4 LAIV organoid stained for markers of T follicular helper cells. For organoid cultures (b-e), representative examples are shown from organoid cultures derived from three different donors. 
a

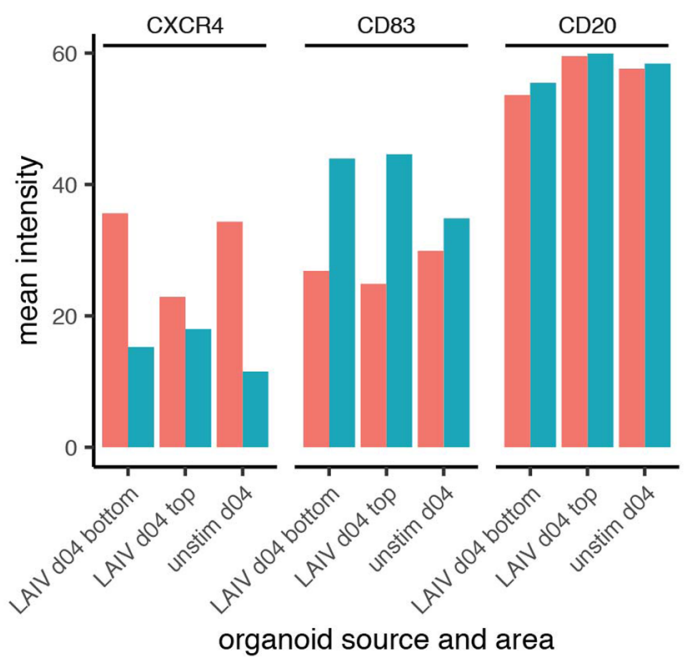

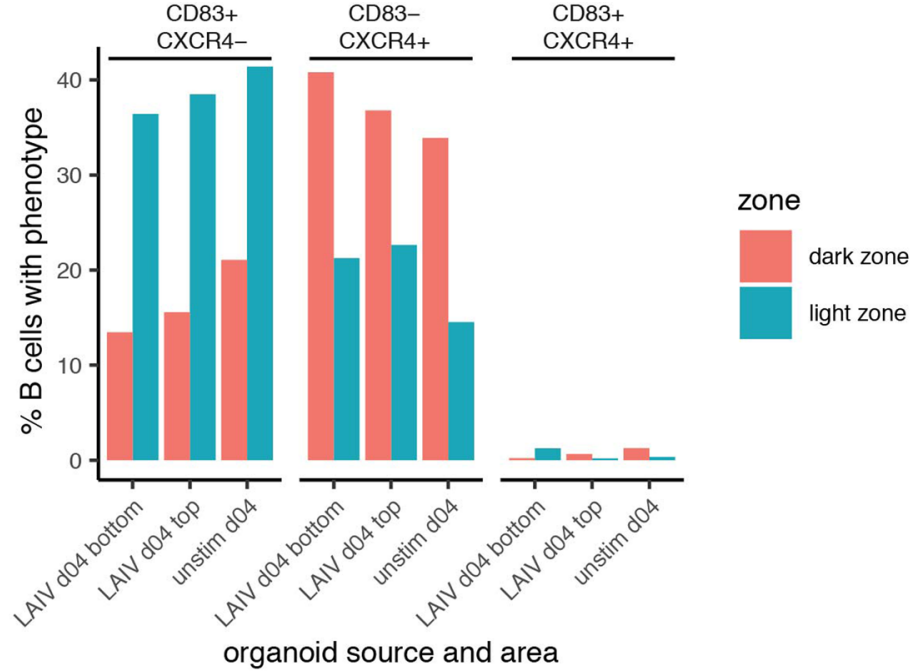

day 4 LAIV-stimulated organoid

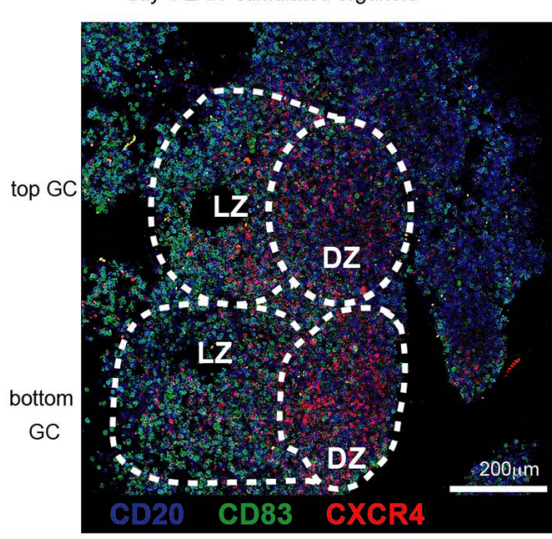

day 4 unstimulated organoid

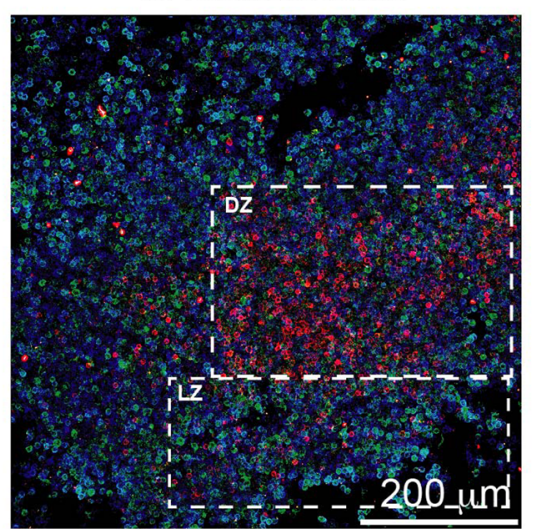

b

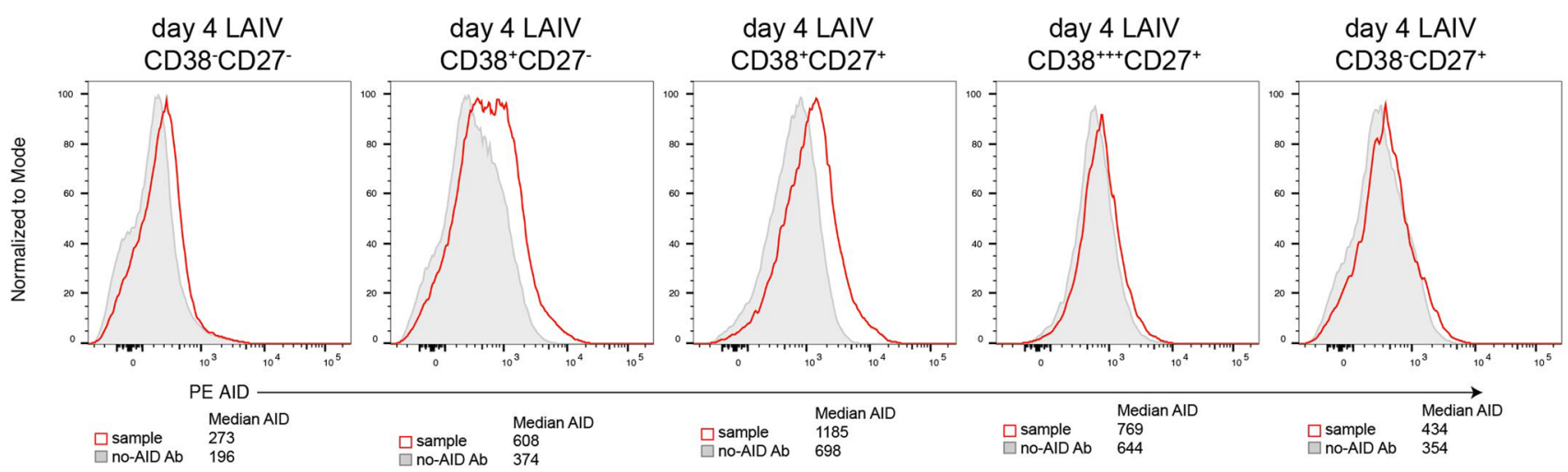

Extended Data Fig. 3 | Quantification of organoid organization and function. a, Quantification of CXCR4 and CD83 expression levels (mean intensity, left panel) and percent positive (percentage of CD20+B cells, right panel) in day 4 tonsil organoids from one donor. Two areas of an LAIV-stimulated organoid (top GC and bottom GC as also shown in Fig. 3a) and a representative GC-organized area from an unstimulated organoid were used for the calculation. b, Representative intracellular AID flow cytometry staining profiles. Data shown are from a day 4, LAIV-stimulated organoid culture. Total B cells were subsetted based on CD38 and CD27 expression and are shown as individual profiles in red compared to a 'no AID primary antibody' FMO control in grey. 
a

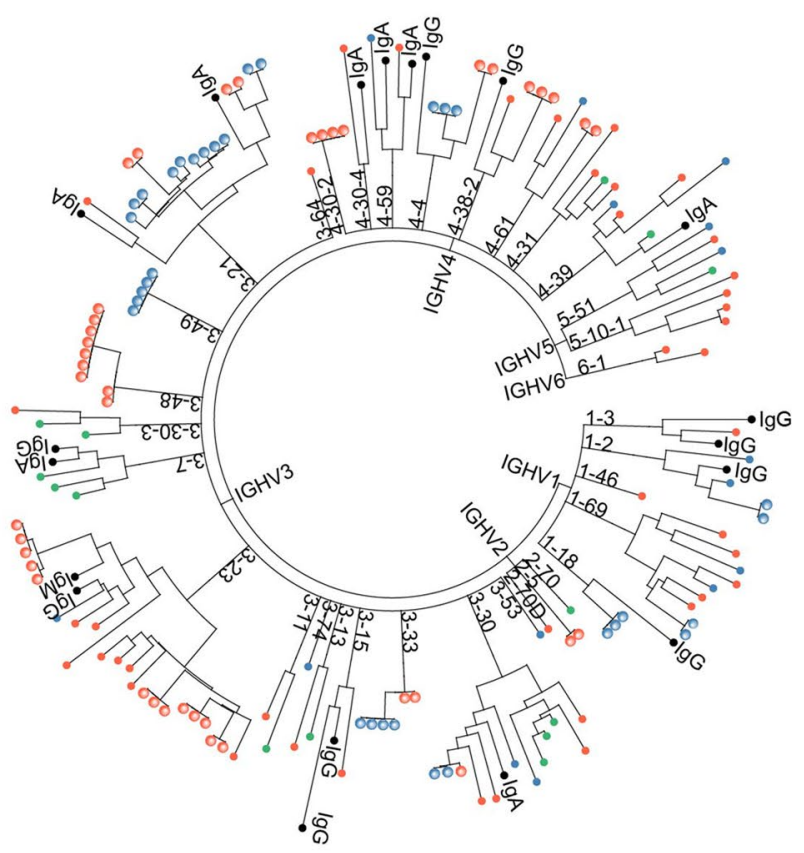

b
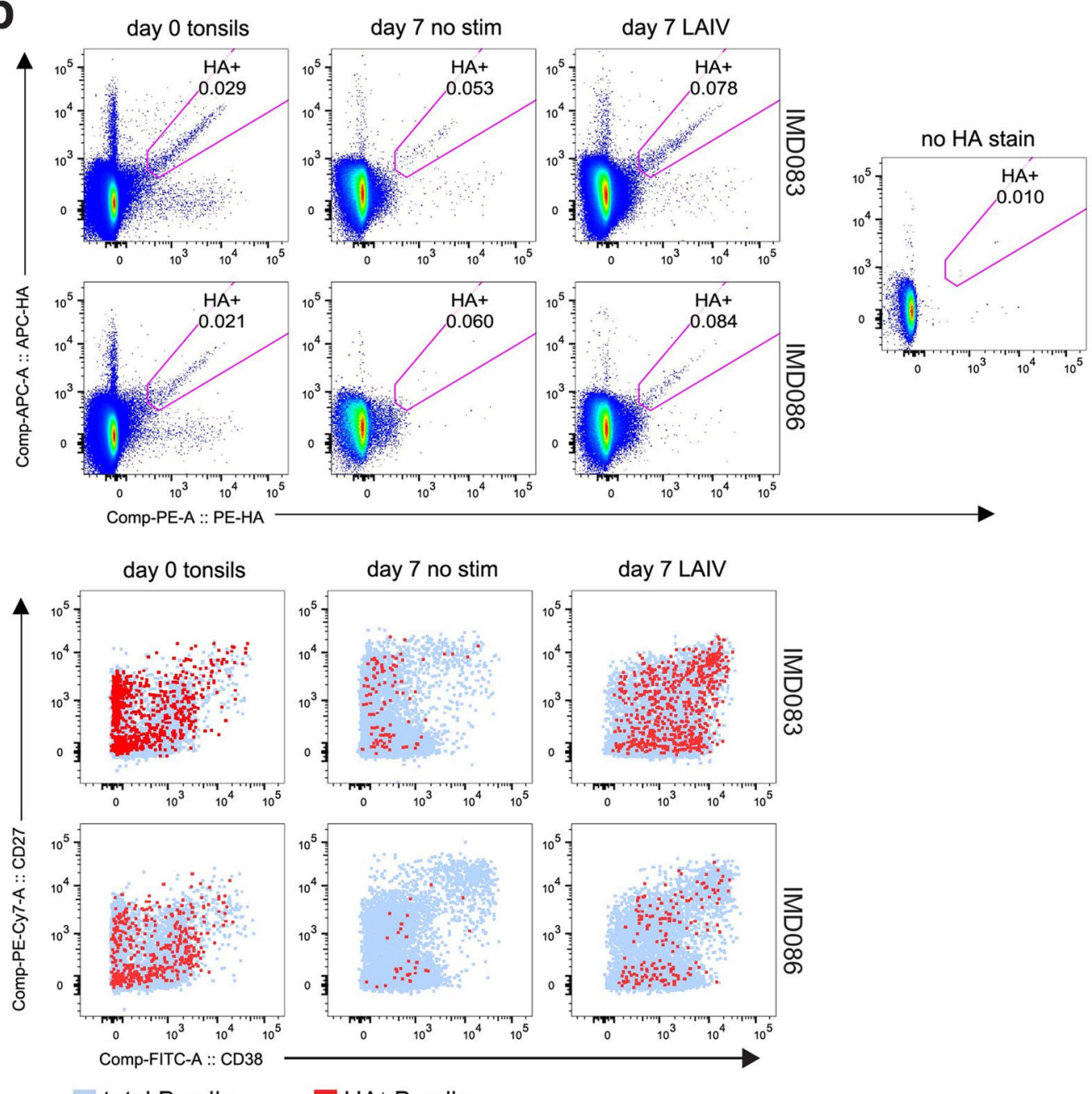

total $B$ cells

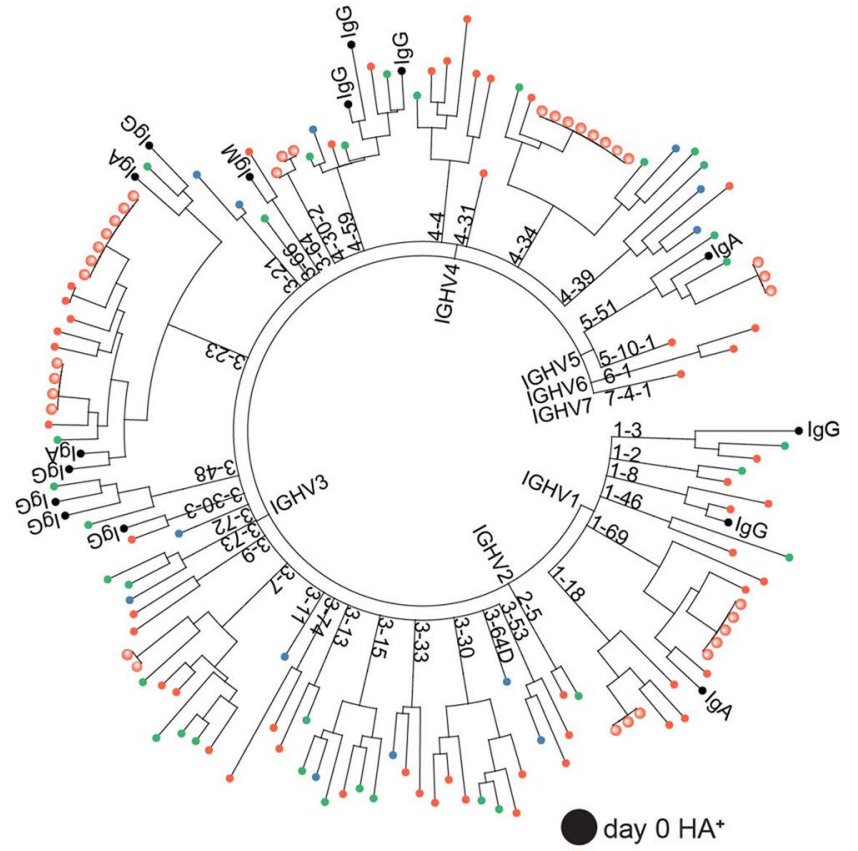

day $7 \mathrm{HA}^{+} \operatorname{lgg}$

day $7 \mathrm{HA}^{+} \operatorname{IgM}$

day $7 \mathrm{HA}^{+} \operatorname{lgA}$

000 clonal families

Extended Data Fig. 4 | See next page for caption. 
Extended Data Fig. 4 | B cell receptor sequencing from HA-specific B cells. a, Phylogenetic trees from two donors are shown (top). Day 0 clones are indicated by black points. Different isotypes ( $\lg M, \lg A, \lg G$ ) are indicated by color (green, blue, and red respectively). Oligoclonal populations and clonal families from single cell data are represented by larger points (open circles). Clonal families were defined as BCR sequences that use the same $V$ and J genes and have at least 70\% amino acid identity in the CDR3 region for heavy and light chains. $\mathbf{b}$, FACS staining and B cell phenotypes of HA-specific B cells compared to the total B cell pool. 


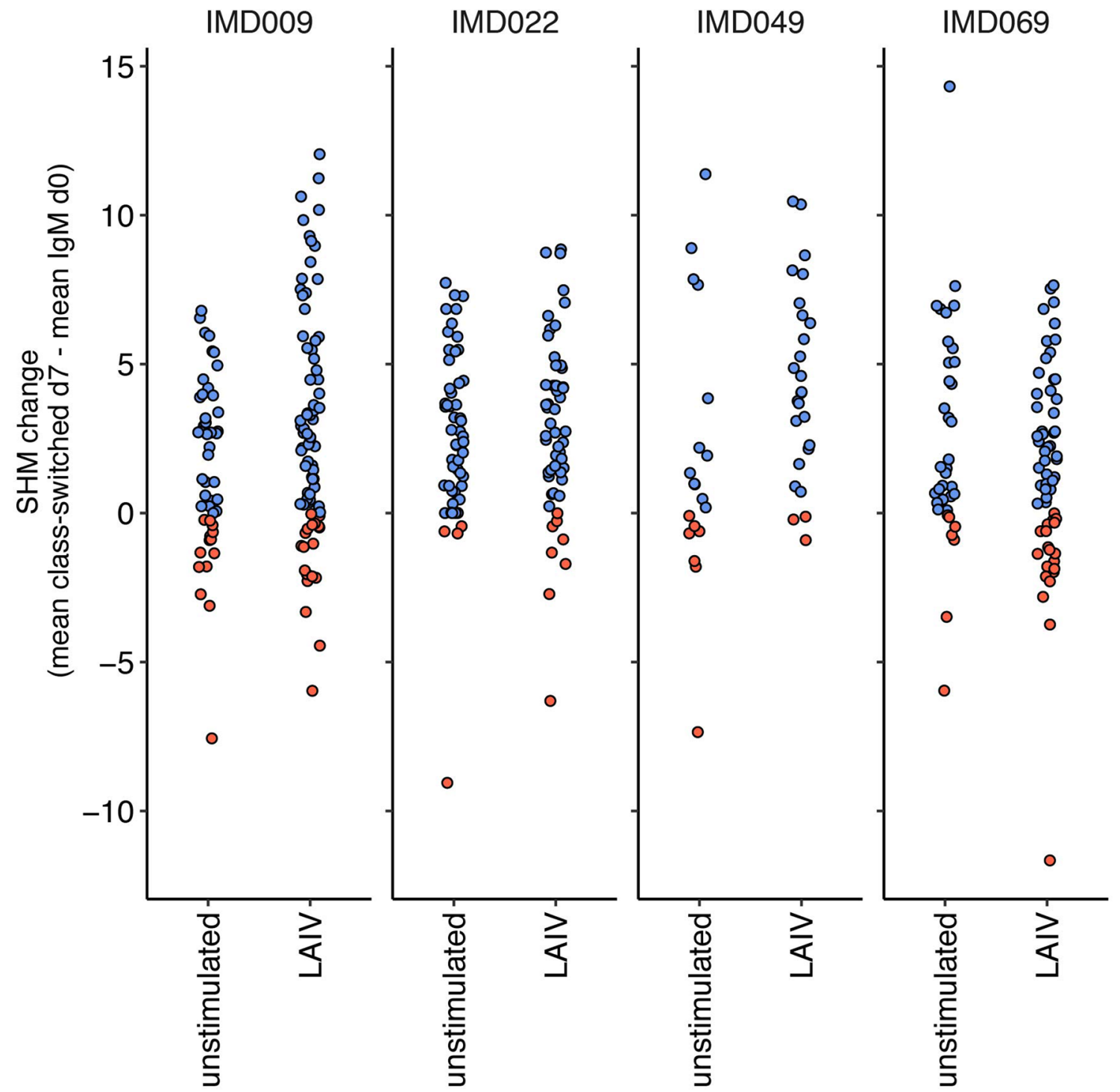

Extended Data Fig. 5 | Tracking individual heavy-chain BCR lineages before and after LAIV stimulation in tonsil organoids show evidence of isotype switching. Immunoglobulin heavy-chain gene rearrangements for each isotype were sequenced from total memory B cells, germinal center B cells, and plasmablasts sorted from cultures of four donors on days 0 and 7 . Heavy-chain BCR sequences from lineages that contained members as only lgM on day $\mathrm{O}$ and as only isotype-switched on day 7 were compared for their somatic hypermutation levels. For each lineage, the mean SHM was calculated for day 0 IgM members and for day 7 switched members, and the difference between these means was plotted. Lineages with increased mutation are shown in blue and those with decreased mutation in red. 


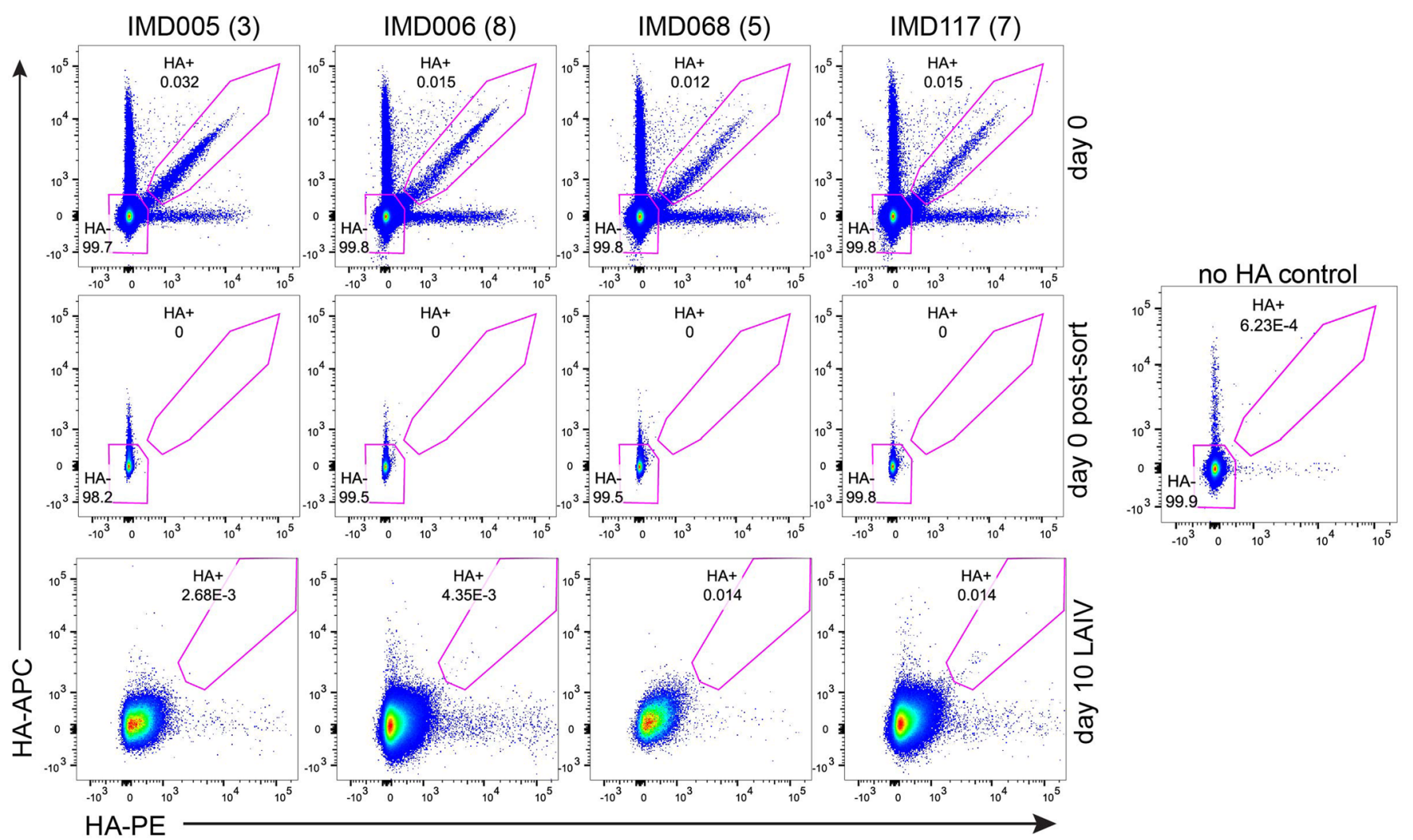

Extended Data Fig. 6 | Depletion of pre-existing HA-specific and non-naive B cells do not prevent production of new high-affinity HA ${ }^{+}$B cells after organoid culture. B cells with high affinity BCRs for A/California $2009 \mathrm{HA}^{+}$and all non-naive $B$ cells were depleted by FACS and depletion was confirmed by post-sort analysis. After 10 days in organoid culture, cells were harvested, re-stained for $A / C a l i f o r n i a ~ H A+B$ cells, and run on a flow cytometer. $n=4$ donors were tested. 
2
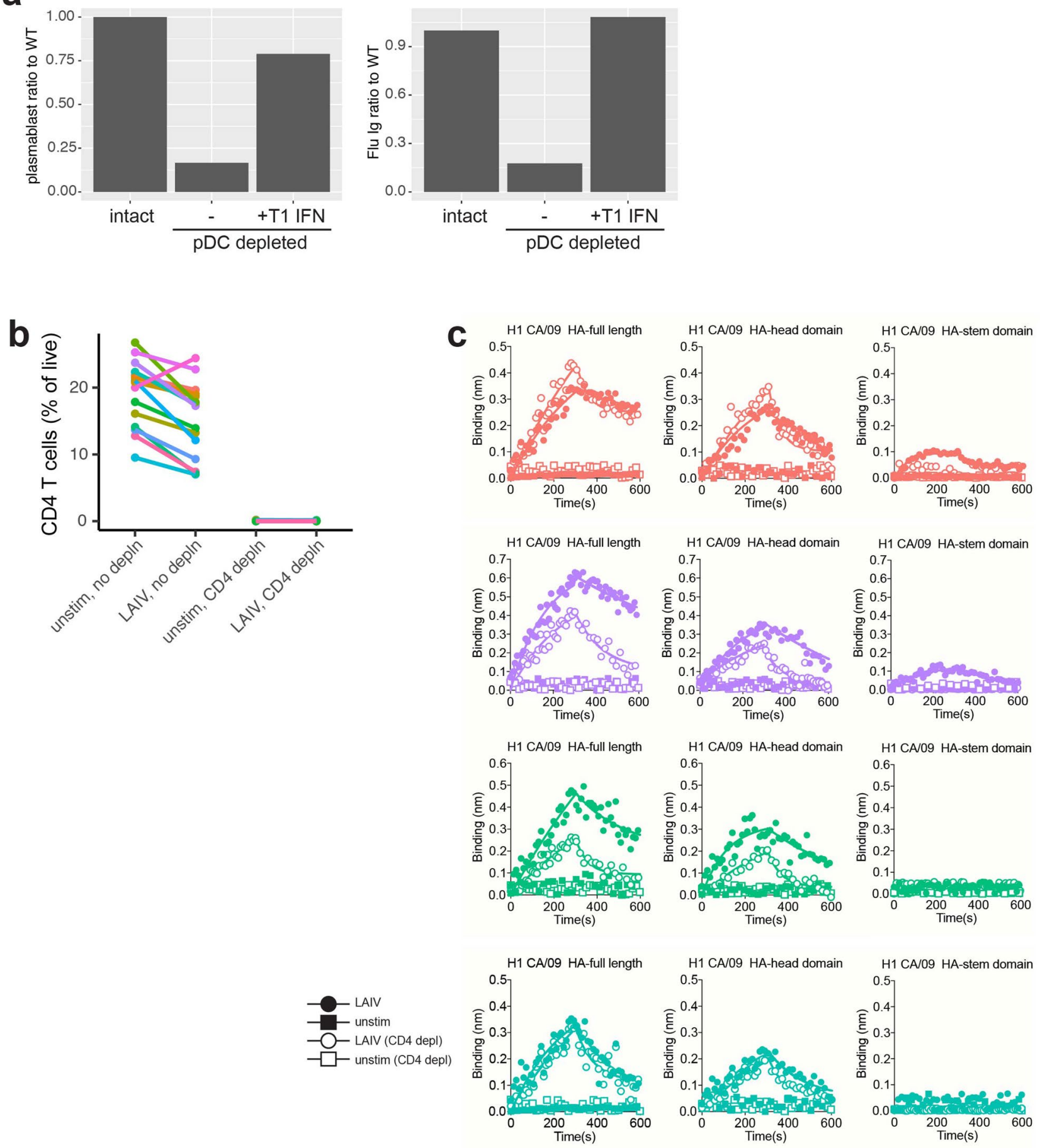

Extended Data Fig. 7 | Effects of cell depletion on influenza-specific antibodies and their affinities. a, Rescue of plasmablast and Ab responses to LAIV stimulation by supplementing pDC-depleted cultures with type I IFNs. b. Frequency of CD4+ T cells (of live cells) in intact and CD4-depleted cultures on day 7. $C D 4+$ cells were depleted by positive selection with magnetic particles. c, Biolayer interferometry data indicating Ab affinity for A/California 2009 H1N1 HA full length, head-specific, and stem domains. Colors are matched to patient samples in (b). 

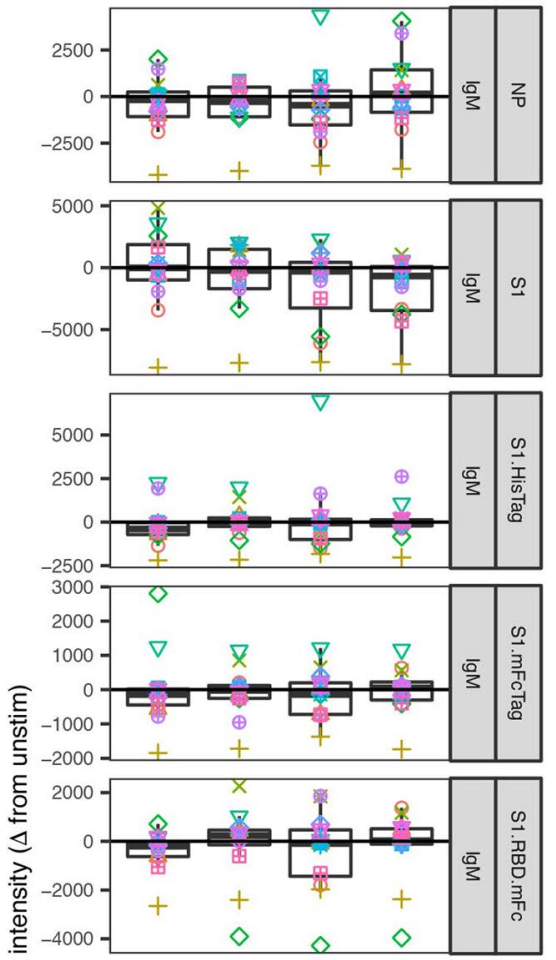

$\frac{\overline{0}}{\frac{\pi}{5}}$
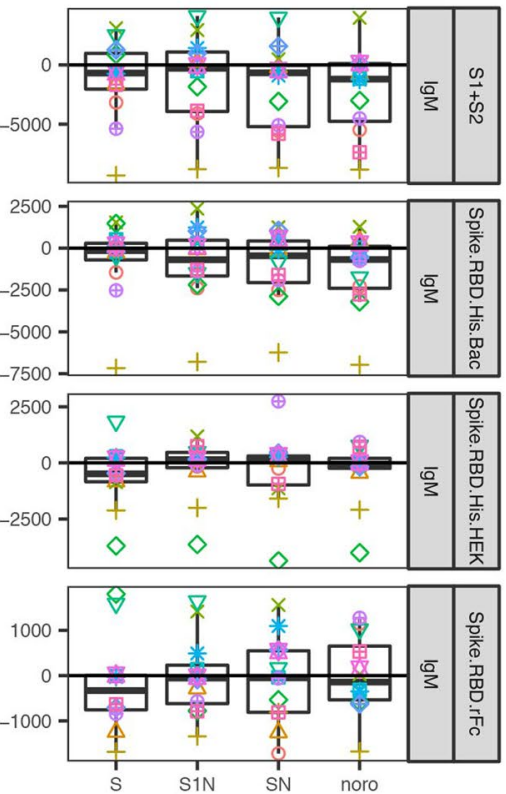
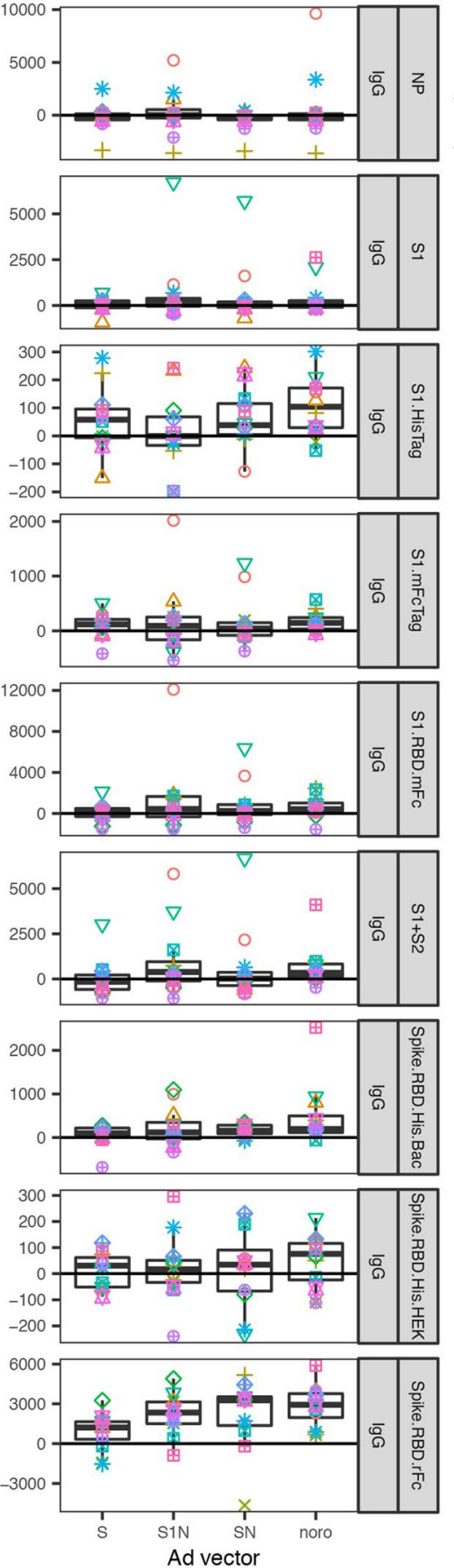
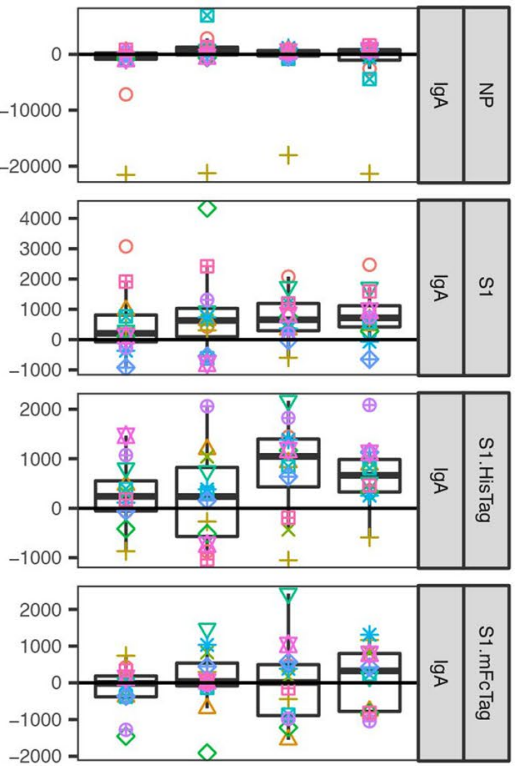

donor

○. IMD019

$\triangle \quad$ IMD079

$+\quad$ IMD117

$\times \quad$ IMD120
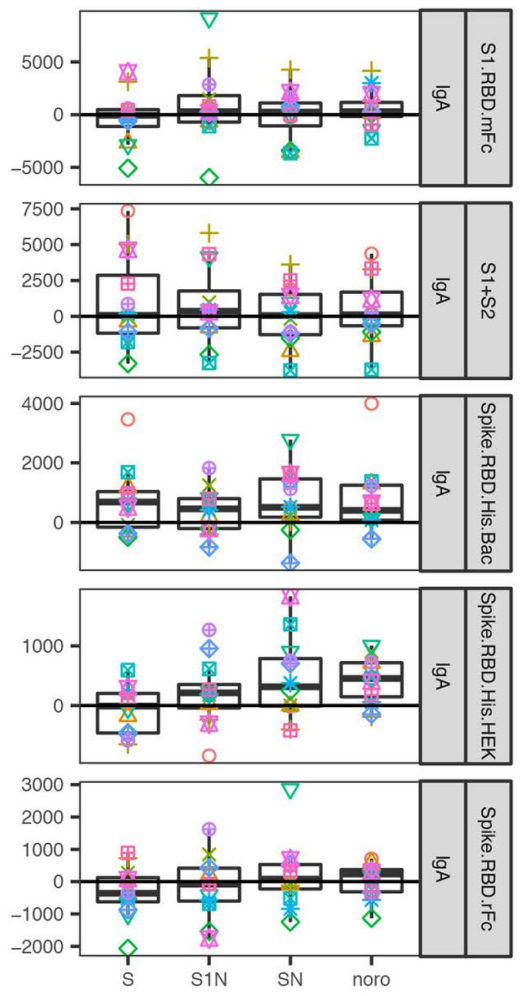

Extended Data Fig. 8 | SARS-CoV2-specific Abs detected in organoid cultures stimulated with Ad5 vectored vaccine candidates. Day 14 post-stimulation culture supernatants were tested for the presence of SARS-CoV2-specific IgM, IgG, and IgA in $n=12$ donors. Abs were detected using a protein microarray. Individuals donors are represented by symbols. The signal intensities shown were background subtracted based on unstimulated control cultures from the same donor. Boxplots show median values with hinges representing first and third quartiles and whiskers representing the highest and lowest value that is within 1.5X the interquartile range of the hinges. 


\section{Reporting Summary}

Nature Research wishes to improve the reproducibility of the work that we publish. This form provides structure for consistency and transparency in reporting. For further information on Nature Research policies, see our Editorial Policies and the Editorial Policy Checklist.

\section{Statistics}

For all statistical analyses, confirm that the following items are present in the figure legend, table legend, main text, or Methods section.

$\mathrm{n} / \mathrm{a}$ Confirmed

$\bigotimes$ The exact sample size $(n)$ for each experimental group/condition, given as a discrete number and unit of measurement

$\bigotimes$ A statement on whether measurements were taken from distinct samples or whether the same sample was measured repeatedly

The statistical test(s) used AND whether they are one- or two-sided

Only common tests should be described solely by name; describe more complex techniques in the Methods section.

$\bigotimes$ A description of all covariates tested

\A description of any assumptions or corrections, such as tests of normality and adjustment for multiple comparisons

$\triangle$ A full description of the statistical parameters including central tendency (e.g. means) or other basic estimates (e.g. regression coefficient)

AND variation (e.g. standard deviation) or associated estimates of uncertainty (e.g. confidence intervals)

For null hypothesis testing, the test statistic (e.g. $F, t, r$ ) with confidence intervals, effect sizes, degrees of freedom and $P$ value noted Give $P$ values as exact values whenever suitable.

Х $\square$ For Bayesian analysis, information on the choice of priors and Markov chain Monte Carlo settings

$\bigotimes \square$ For hierarchical and complex designs, identification of the appropriate level for tests and full reporting of outcomes

$\triangle \square$ Estimates of effect sizes (e.g. Cohen's d, Pearson's $r$ ), indicating how they were calculated

$$
\text { Our web collection on statistics for biologists contains articles on many of the points above. }
$$

\section{Software and code}

Policy information about availability of computer code

Data collection Sequencing data were collected with Illumina platforms and debarcoded using pipelines previously reported. The original references are cited in-text.

Data analysis Data were analyzed using R version 3.5.0. Imaging was analyzed with ImageJ version 2.0.0. Flow cytometry data were analyzed with Flowjo version 10.7.0.

For manuscripts utilizing custom algorithms or software that are central to the research but not yet described in published literature, software must be made available to editors and

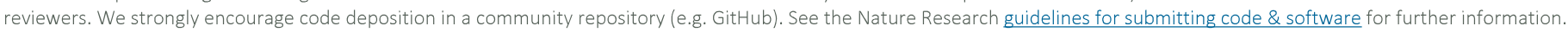

\section{Data}

Policy information about availability of data

All manuscripts must include a data availability statement. This statement should provide the following information, where applicable:

- Accession codes, unique identifiers, or web links for publicly available datasets

- A list of figures that have associated raw data

- A description of any restrictions on data availability

Requests for raw data, analyzed data, and materials will be reviewed by Stanford University to determine if the request is subject to intellectual property or confidentiality obligations. Data and materials that can be shared will be released using a Material Transfer Agreement. Targeted single-cell RNAseq data are available in the Gene Expression Omnibus (GEO) at GSE158459. 
Please select the one below that is the best fit for your research. If you are not sure, read the appropriate sections before making your selection.

\ Life sciences

Behavioural \& social sciences Ecological, evolutionary \& environmental sciences

For a reference copy of the document with all sections, see nature.com/documents/nr-reporting-summary-flat.pdf

\title{
Life sciences study design
}

All studies must disclose on these points even when the disclosure is negative.

$\begin{array}{ll}\text { Sample size } & \begin{array}{l}\text { Sample sizes varied from } n=1 \text { to } n=16 \text { depending on the experiments performed. Since portions of the data rely upon limited clinical samples, } \\ \text { these tissues were used upon availability. In experiments with larger numbers, we incorporated samples from both sexes and across ages. }\end{array} \\ \text { Data exclusions } & \text { No data excluded. } \\ \text { Replication } & \begin{array}{l}\text { Numerous donors were tested as indicated in-text. Instances where some subjects responded and others did not are also indicated in-text. } \\ \text { We expect inter-donor variation in the ability to respond to the antigens tested. We used different donors to show the distribution of possible } \\ \text { responses and did not intentionally replicate intra-donor experiments. }\end{array}\end{array}$

Randomization Experimental groups were age-related or stimulation-related, thus randomization N/A.

Blinding Age-based analyses were performed post-data collection. Researchers were blinded to donor age during data collection and gating analysis.

\section{Reporting for specific materials, systems and methods}

We require information from authors about some types of materials, experimental systems and methods used in many studies. Here, indicate whether each material, system or method listed is relevant to your study. If you are not sure if a list item applies to your research, read the appropriate section before selecting a response.

\begin{tabular}{l|l} 
Materials \& experimental syste \\
\hline $\mathrm{n} / \mathrm{a}$ & Involved in the study \\
\hline & $\bigotimes$ Antibodies \\
\hline & $\square$ Eukaryotic cell lines \\
$\square$ & $\square$ Palaeontology and archaeology \\
$\square$ & $\square$ Human research participants \\
$\square$ & $\square$ Clinical data
\end{tabular}

\begin{tabular}{l|l} 
Methods \\
\hline n/a & Involved in the study \\
$\square$ & $\square$ ChIP-seq \\
$\square$ & $\bigotimes$ Flow cytometry \\
$\square$ & $\square$ MRI-based neuroimaging
\end{tabular}

\section{Antibodies}

Antibodies used

\author{
FITC CD138 (DL-101; 352303) 1/100 \\ FITC CD116 (4H1; 305906) 1/100 \\ FITC CD21 (Bu32; 354909) 1/50 \\ FITC CXCR5 (J25D4; 356913) 1/33 \\ AX488 CXCR5 (J25D4; 356911) 1/33 \\ PerCP-CY5.5 CD8 (RPA-T8; 301031) 1/100 \\ PerCP-Cy5,5 CD33 (WM53; 303413) 1/100 \\ PE CD19 (HIB19; 302207) 1/100 \\ PE CD56 (HCD56; 318305) 1/100 \\ PE gd TCR (B1; 331209) 1/100 \\ PE-Cy7 CD27 (O323; 302837) 1/100 \\ PE-CY7 CD123 (6H6; 306009) 1/50 \\ PE-CY7 CD8 (SK1; 344711) 1/100 \\ APC CD38 (HIT2; 303509) 1/200 \\ APC HLA-DR (L243; 307609) $1 / 100$ \\ APC CD27 (O323; 302809) 1/200 \\ Ax700 CD45 (HI30; 304023) 1/100 \\ Ax700 CD14 (M5E2; 301822) 1/100 \\ APC-Cy7 IgD (IA6-2; 348217) 1/50 \\ APC-Cy7 CD16 (3G8; 302017) 1/100 \\ APC-Cy7 CD45RA (HI100; 304127) 1/100 \\ Pac Blue HLA-DR (L243; 307623) 1/100
}


Pac Blue PD-1 (EH12.2H7; 329915) 1/50

BV605 CD3 (OKT3; 317321) 1/100

BV650 CD4 (OKT4; 317435) 1/100

BV650 CD19 (HIB19; 302237) 1/100

BUV395 IgM (BD; G20-127; 563903) $1 / 20$

BUV395 CD45RA (BD; HI100; 740298) 1/20

AID (eBioscience; mAID-2; 13-5959-82) 1/100

Validation

All antibodies are commercially available and have been validated by the manufacturer for use in flow cytometry. Please consult the corresponding catalogue numbers provided above for the manufacturer validation data on their respective websites. All antibodies were titrated for our experiments prior to use with organoid culture samples. For AID staining, we confirmed specificity with a noprimary-antibody control.

\section{Human research participants}

\section{Policy information about studies involving human research participants}

Population characteristics

Children undergoing tonsillectomy were recruited with IRB approval for donating tissues to this study - $80 \%$ for obstructive sleep apnea, $17 \%$ for recurrent tonsillitis, and 3\% for sleep-disordered breathing. Patient ages ranged from $2-16$ years. $44 \%$ of participants were female, $56 \%$ male.

Donor lung lymph nodes and spleens were provided by the Gift of Hope Organ and Tissue Donor Network to the University of Chicago. These tissues were determined to have IRB Exempt Status by the University of Chicago IRB. Only de-identified demographic information was obtained.

Recruitment

Patients were recruited immediately prior to surgery. Patients or their guardians who could not provide explicit written informed consent were excluded. Tonsil patients with serious infections or who were taking systemic immunomodulatory drugs were excluded from the study. All living participants in this study provided written informed consent (or through their legal guardians). Individuals responsible for consenting patients and collecting tissues were not involved in the scientific aspects of the study. We do not expect any systematic bias in our collection strategy.

Ethics oversight

IRB for Stanford University (tonsils; written informed consent from patients or their legal guardians; approval numbers 30837 and 47690) and the University of Chicago (LNs and spleens, exempt status)

Note that full information on the approval of the study protocol must also be provided in the manuscript.

\section{Flow Cytometry}

Plots

Confirm that:

\The axis labels state the marker and fluorochrome used (e.g. CD4-FITC).

$\bigotimes$ The axis scales are clearly visible. Include numbers along axes only for bottom left plot of group (a 'group' is an analysis of identical markers).

\All plots are contour plots with outliers or pseudocolor plots.

$\bigotimes$ A numerical value for number of cells or percentage (with statistics) is provided.

\section{Methodology}

Sample preparation

Instrument

Software

Cell population abundance

Gating strategy

$\bigotimes$ Tick this box to confirm that a figure exemplifying the gating strategy is provided in the Supplementary Information.
The tissue processing is a major component of the technique and thus is extensively described in the methods. After isolation of tonsil cells and culture, samples were prepared as follows: tonsil organoids were harvested from the upper portion of transwells by rinsing the membranes with PBS. Cells were washed with FACS buffer (PBS + 0.1\% BSA, $0.05 \%$ sodium azide, $2 \mathrm{mM}$ EDTA) and stained with the anti-human antibodies in the presence of Fc block with live/dead aqua zombie stain. Cells were washed and then resuspended in FACS buffer prior to analysis.

LSRII (4-laser system, BD), Ariall or Fusion sorter (5-laser system, BD)

FACS DIVA (BD)

Samples were sorted for depletion and reconstituted at physiologically relevant, pre-existing frequencies as controls. Postsort analysis was performed as described in-text.

Gating strategy is provided in extended data. Briefly, cells were identified based on scatter properties, singlets based on FSC$\mathrm{H}$ vs A, live cells, CD45+ vs CD45- cells, and lymphocyte populations based on established markers shown. 